Discrete ANALYSis, 2020:13, 34 pp.

www.discreteanalysisjournal.com

\title{
Sylvester-Gallai type theorems for quadratic polynomials
}

\author{
Amir Shpilka * \\ Received 26 June 2019; Revised 2 April 2020; Published 12 August 2020
}

\begin{abstract}
We prove Sylvester-Gallai type theorems for quadratic polynomials. Specifically, we prove that if a finite collection $Q$, of irreducible polynomials of degree at most 2 , satisfy that for every two polynomials $Q_{1}, Q_{2} \in \mathcal{Q}$ there is a third polynomial $Q_{3} \in \mathcal{Q}$ so that whenever $Q_{1}$ and $Q_{2}$ vanish then also $Q_{3}$ vanishes, then the linear span of the polynomials in $Q$ has dimension $O(1)$. We also prove a colored version of the theorem: If three finite sets of quadratic polynomials satisfy that for every two polynomials from distinct sets there is a polynomial in the third set satisfying the same vanishing condition then all polynomials are contained in an $O(1)$-dimensional space.

This answers affirmatively two conjectures of Gupta [Gup14] that were raised in the context of solving certain depth-4 polynomial identities.

To obtain our main theorems we prove a new result classifying the possible ways that a quadratic polynomial $Q$ can vanish when two other quadratic polynomials vanish. Our proofs also require robust versions of a theorem of Edelstein and Kelly (that extends the Sylvester-Gallai theorem to colored sets).
\end{abstract}

Key words and phrases: Sylvester-Gallai theorem, quadratic polynomials, polynomial identity testing

\section{Introduction}

The Sylvester-Gallai theorem asserts that if a finite set of points has the property that every line passing through any two points in the set also contains a third point in the set then all the points in the set are colinear. Many variants of this theorem were studied: extensions to higher dimensions, colored versions,

\footnotetext{
${ }^{*}$ The research leading to these results has received funding from the Israel Science Foundation (grant number 552/16) and from the Len Blavatnik and the Blavatnik Family foundation
} 


\section{AMIR SHPILKA}

robust versions and many more. For a survey on the Sylvester-Gallai theorem and its variants see [BM90]. One specific extension that is relevant to our work is the following colored version that was obtained by Edelstein and Kelly: If three finite sets of points satisfy that every line passing through points from two different sets also contains a point from the third set, then, in this case too all the points belong to a low dimensional space.

Another extension of the theorem that is relevant to our work was proved in [BDWY13, DSW14]. There the authors proved the following robust version of the Sylvester-Gallai theorem (along with other robust versions of similar theorems): if a finite set of points satisfies that for every point $p$ in the set there is a $\delta$ fraction of other points so that for each of them, the line passing through it and $p$, spans a third point in the set, then the set is contained in an $O(1 / \delta)$-dimensional space.

While these theorems may seem unrelated to computation at first sight they have important consequences for locally decodable and locally correctable codes [BDWY13, DSW14], for reconstruction of certain depth-3 circuits [Shp09, KS09a, Sin16] and for the polynomial identity testing (PIT for short) problem, which we describe next.

The PIT problem asks to give a deterministic algorithm that given arithmetic circuit as input determines whether it computes the identically zero polynomial. This is a fundamental problem in theoretical computer science that has attracted a lot of attention both because of its intrinsic importance, its relation to other derandomization problems [KSS15, Mul17, FS13, FGT19, GT17, ST17] and its connections to lower bounds for arithmetic circuits [HS80, Agr05, KI04, DSY09, FSV18, CKS18]. For more on the PIT problem see [SY10, Sax09, Sax14, For14].

The case most relevant to Sylvester-Gallai type theorems is when the input circuit is a depth-3 circuit with small top fan-in. Specifically, a homogeneous $\Sigma^{[k]} \Pi^{[d]} \Sigma$ circuit in $n$ variables computes a polynomial of the following form

$$
\Phi\left(x_{1}, \ldots, x_{n}\right)=\sum_{i=1}^{k} \prod_{j=1}^{d} \ell_{i, j}\left(x_{1}, \ldots, x_{n}\right),
$$

where each $\ell_{i, j}$ is a linear form. Consider the PIT problem for $\Sigma^{[3]} \Pi^{[d]} \Sigma$ circuits. I.e., $\Phi$ is given as in Equation 1 and it has 3 multiplication gates, i.e. $k=3$. If $\Phi$ computes the zero polynomial then we have, for every $j, j^{\prime} \in[d]$, that

$$
\prod_{i=1}^{d} \ell_{1, i} \equiv 0 \quad \bmod \ell_{2, j}, \ell_{3, j^{\prime}}
$$

As the zero set of two linear functions is an irreducible variety, we get as a consequence that for every $j, j^{\prime} \in[d]$, the linear functions $\ell_{2, j}$ and $\ell_{3, j^{\prime}}$ span a linear function in $\left\{\ell_{1,1}, \ldots, \ell_{1, d}\right\}$. In other words, the three sets $\mathcal{T}_{i}=\left\{\ell_{i, 1}, \ldots, \ell_{i, d}\right\}$, for $i \in\{1,2,3\}$, satisfy the conditions of the Edelstein-Kelly theorem described above, ${ }^{1}$ and hence span a low dimensional space. Thus, if $\Phi \equiv 0$ then we can rewrite the expression for $\Phi$ using only constantly many variables (after a suitable invertible linear transformation). This allows efficient PIT algorithms for such $\Sigma^{[3]} \Pi^{[d]} \Sigma$ circuits. The case of more than 3 multiplication gates is more complicated and satisfies a similar higher dimensional condition. This rank-bound approach for PIT of $\Sigma \Pi \Sigma$ circuits was raised in [DS07] and later carried out in [KS09b, SS13]. ${ }^{2}$

\footnotetext{
${ }^{1}$ The theorem speaks about line through points rather than span of vectors, but it is not hard to see how to translate the Edelstein-Kelly theorem to this setting as well. See Remark 17.

${ }^{2}$ The best algorithm for PIT of $\Sigma^{[k]} \Pi^{[d]} \Sigma$ circuits was obtained through a different, yet related, approach in [SS12].
} 
While such rank-bounds found important applications in studying PIT of depth-3 circuits, it seemed that such an approach cannot work for depth- $4 \Sigma \Pi \Sigma \Pi$ circuits, ${ }^{3}$ even in the simplest case where there are only 3 multiplication gates and the bottom fan-in is two, i.e., for homogeneous $\Sigma^{[3]} \Pi^{[d]} \Sigma \Pi^{[2]}$ circuits that compute polynomials of the form

$$
\Phi\left(x_{1}, \ldots, x_{n}\right)=\prod_{j=1}^{d} Q_{1, j}\left(x_{1}, \ldots, x_{n}\right)+\prod_{j=1}^{d} Q_{2, j}\left(x_{1}, \ldots, x_{n}\right)+\prod_{j=1}^{d} Q_{3, j}\left(x_{1}, \ldots, x_{n}\right),
$$

where each $Q_{i . j}$ is a homogeneous quadratic polynomial. Indeed, we if try to reason as before then we get

$$
\prod_{j=1}^{d} Q_{1, j}\left(x_{1}, \ldots, x_{n}\right)=0 \quad \bmod Q_{2, j}, Q_{3, j^{\prime}}
$$

However, unlike the linear case it is not clear what can be concluded now. Indeed, if a product of linear functions vanishes modulo two linear functions, then we know that one function in the product must be in the linear span of those two linear functions. For quadratic polynomials this is not necessarily the case. For example, note that if for a quadratic $Q$ we have that $Q=0$ and $Q+x^{2}=0$ then also $Q+x y=0$, and, clearly, we can find $Q$ such that $Q+x y$ is not spanned by $Q$ and $Q+x^{2}$. An even more problematic difference is that it may be the case that Equation 3 holds but that no $Q_{1, j}$ always vanishes when, say, $Q_{2,1}, Q_{3,1}$ vanish. For example, let

$$
Q_{1}=x y+z w \quad, \quad Q_{2}=x y-z w \quad, \quad Q_{3}=x w \quad, \quad Q_{4}=y z .
$$

Then, it is not hard to verify that

$$
Q_{3} \cdot Q_{4} \equiv 0 \quad \bmod Q_{1}, Q_{2} .
$$

but neither $Q_{3}$ nor $Q_{4}$ vanish identically modulo $Q_{1}, Q_{2}$. Thus, the PIT problem for sums of products of quadratics seem much harder than the corresponding problem for depth-3 circuits. Indeed, currently no efficient deterministic PIT algorithm is known for $\Sigma^{[3]} \Pi^{[d]} \Sigma \Pi^{[2]}$ circuits.

In spite of the above, Beecken et al. [BMS13, Gup14] and Gupta [Gup14] conjectured that perhaps the difference between the quadratic case and the linear case is not so dramatic. In fact, they suggested that this may be the case for any constant degree and not just for quadratics. Specifically, Gupta observed that whenever Equation 3 holds it must be the case that there are four polynomials in $\left\{Q_{1, j}\right\}$ whose product vanishes identically. That is, for every $\left(j, j^{\prime}\right) \in[d]^{2}$ there are $i_{1, j, j^{\prime}}, i_{2, j, j^{\prime}}, i_{3, j, j^{\prime}}, i_{4, j, j^{\prime}} \in[d]$ so that

$$
Q_{1, i_{1, j, j^{\prime}}} \cdot Q_{1, i_{2, j, j^{\prime}}} \cdot Q_{1, i_{3, j, j^{\prime}}} \cdot Q_{1, i_{4, j, j^{\prime}}} \equiv 0 \quad \bmod Q_{2, j}, Q_{3, j^{\prime}} \cdot
$$

Gupta then raised the conjecture that whenever this holds for every $j, j^{\prime}$ and for every two of the multiplication gates, then it must be the case that the algebraic rank of the set $\left\{Q_{i, j}\right\}$ is $O(1)$. More generally, Gupta conjectured that this is the case for any fixed number of sets.

\footnotetext{
${ }^{3}$ Though we note that for multilinear $\Sigma \Pi \Sigma \Pi$ circuits Saraf and Volkovich obtained an analogous bound on the sparsity of the polynomials computed by the multiplication gates in a zero circuit [SV18].
} 
AMIR SHPILKA

Conjecture 4 (Conjecture 1 in [Gup14]). Let $\mathcal{F}_{1}, \ldots, \mathcal{F}_{k}$ be finite sets of irreducible homogenous polynomials in $\mathbb{C}\left[x_{1}, \ldots, x_{n}\right]$ of degree $\leq r$ such that $\cap_{i} \mathcal{F}_{i}=\emptyset$ and for every $k-1$ polynomials $Q_{1}, \ldots, Q_{k-1}$, each from a distinct set, there are $P_{1}, \ldots, P_{c}$ in the remaining set such that whenever $Q_{1}, \ldots, Q_{k-1}$ vanish then also the product $\prod_{i=1}^{c} P_{i}$ vanishes. Then, $\operatorname{trdeg}_{\mathbb{C}}\left(\cup_{i} \mathcal{F}_{i}\right) \leq \lambda(k, r, c)$ for some function $\lambda$, where trdeg stands for the transcendental degree (which is the same as algebraic rank).

The condition in the conjecture can be stated equivalently as

$$
\prod_{i=1}^{c} P_{i} \in \sqrt{\left(Q_{1}, \ldots, Q_{k-1}\right)}
$$

where the object on the right hand side is the radical of the ideal generated by $\left\{Q_{i}\right\}_{i=1}^{k-1}$ (see subsection 2.1). Note that for $r=1$ we have also $c=1$ and by the Edelstein-Kelly theorem $\lambda$ is $\leq 2$ in this case (and we can replace algebraic rank with linear rank).

In [BMS13] Beecken et al. conjectured that the algebraic rank of simple and minimal $\Sigma^{[k]} \Pi^{[d]} \Sigma \Pi^{[r]}$ circuits (see their paper for definition of simple and minimal) is $O_{k}(\log d)$. We note that this conjecture is weaker than Gupta's as every zero $\Sigma^{[k]} \Pi^{[d]} \Sigma \Pi^{[r]}$ circuit gives rise to a structure satisfying the conditions of Gupta's conjecture, but the other direction is not necessarily true. Beecken et al. also showed how to obtain a deterministic PIT for $\Sigma^{[k]} \Pi^{[d]} \Sigma \Pi^{[r]}$ circuits assuming the correctness of their conjecture.

As an approach towards solving Conjecture 4 Gupta set up a collection of conjectures, each of which is a natural extension of a known Sylvester-Gallai type theorem for the case of higher degree polynomials. The first conjecture is a direct analog of the Sylvester-Gallai theorem where we replace the requirement that a line through two points contains a third with a more algebraic condition: that for every two polynomials there is a third one so that whenever the two polynomials vanish then also the third vanishes.

Conjecture 5 (Conjecture 2 of [Gup14]). Let $Q_{1}, \ldots, Q_{m} \in \mathbb{C}\left[x_{1}, \ldots, x_{n}\right]$ be irreducible and homogenous polynomials of degree $\leq r$ such that for every pair of distinct $Q_{i}, Q_{j}$ there is a distinct $Q_{k}$ so that whenever $Q_{i}$ and $Q_{j}$ vanish then so does $Q_{k}$. Then $\operatorname{trdeg}_{\mathbb{C}}\left(Q_{1}, \ldots, Q_{m}\right) \leq \lambda(r)$.

Note that Sylvester-Gallai's theorem is equivalent to the special case $r=1$. A more general conjecture in [Gup14] is that a similar phenomenon holds when the polynomials come from different sets.

Conjecture 6 (Conjecture 30 of [Gup14]). Let $R, B, G$ be finite disjoint sets of irreducible homogenous polynomials in $\mathbb{C}\left[x_{1}, \ldots, x_{n}\right]$ of degree $\leq r$ such that for every pair $Q_{1}, Q_{2}$ from distinct sets there is a $Q_{3}$ in the remaining set so that whenever $Q_{1}$ and $Q_{2}$ vanish then also $Q_{3}$ vanishes. Then $\operatorname{trdeg}_{\mathbb{C}}(R \cup B \cup G) \leq$ $\lambda(r) .^{4}$

The case $r=1$ is the Edelstein-Kelly theorem. Both Conjecture 5 and Conjecture 6 were open, prior to this work, for any degree $r>1$.

\footnotetext{
${ }^{4}$ Here and in Conjectures 4 and 5 we actually need to assume that the polynomials are pairwise linearly independent.
} 


\subsection{Our Results}

Our main results give affirmative answers to Conjecture 5 and Conjecture 6 for the case $r=2$. This shows that a Sylvester-Gallai type phenomenon holds for degree 2 and we believe this indicates that this might be the case for higher degrees as well. Specifically we prove the following two theorems. The first is an extension of the Sylvester-Gallai theorem to quadratic polynomials. It confirms Conjecture 5 for the case $r=2$.

Theorem 7. Let $\left\{Q_{i}\right\}_{i \in[m]}$ be homogeneous quadratic polynomials over $\mathbb{C}$ such that each $Q_{i}$ is either irreducible or a square of a linear function. Assume further that for every $i \neq j$ there exists $k \notin\{i, j\}$ such that whenever $Q_{i}$ and $Q_{j}$ vanish $Q_{k}$ vanishes as well. Then the linear span of the $Q_{i}$ 's has dimension $O(1)$.

The second theorem is an extension of the theorem of Edelstein-Kelly to quadratic polynomials, which gives an affirmative answer to Conjecture 6 for the case $r=2$.

Theorem 8. Let $\mathcal{T}_{1}, \mathcal{T}_{2}$ and $\mathcal{T}_{3}$ be finite sets of homogeneous quadratic polynomials over $\mathbb{C}$ satisfying the following properties:

- Each $Q \in \cup_{i} \mathcal{T}_{i}$ is either irreducible or a square of a linear function. ${ }^{5}$

- No two polynomials are multiples of each other (i.e., every pair is linearly independent).

- For every two polynomials $Q_{1}$ and $Q_{2}$ from distinct sets there is a polynomial $Q_{3}$ in the third set so that whenever $Q_{1}$ and $Q_{2}$ vanish then also $Q_{3}$ vanishes.

Then the linear span of the polynomials in $\cup_{i} \mathcal{T}_{i}$ has dimension $O(1)$.

Note that what we proved is even stronger than what was conjectured in Conjectures 5 and 6 . There the conjecture is that there is an upper bound on the algebraic rank whereas our results give an upper bound on the linear rank (which of course trivially implies an upper bound on the algebraic rank).

From the perspective of PIT our results do not imply Conjecture 4, even for the case of $k=3$ and $r=2$, yet we believe they are a significant step in the direction of resolving this conjecture and obtaining a PIT algorithm for $\Sigma^{[3]} \Pi^{[d]} \Sigma \Pi^{[2]}$ circuits.

An important tool in the proof of Theorem 7 is a result of [BDWY13, DSW14] that gives a robust version of the Sylvester-Gallai theorem (see subsection 2.2). For the proof of Theorem 8 we need the following relaxation of the Edelstein-Kelly theorem. Roughly, three finite sets form a $\delta$-EK configuration if for every point $p$ in one set a $\delta$ fraction of the points in a second set satisfy that the line connecting each of them to $p$ passes through a point in the third set.

Theorem 9. Let $0<\delta \leq 1$ be any constant. Let $\mathcal{T}_{1}, \mathcal{T}_{2}, \mathcal{T}_{3} \subset \mathbb{C}^{n}$ be disjoint finite subsets that form a $\delta$-EK configuration. Then $\operatorname{dim}\left(\operatorname{span}\left\{\cup_{i} \mathcal{T}_{i}\right\}\right) \leq O\left(1 / \delta^{3}\right)$.

This theorem is similar in nature to the results proved in [BDWY13, DSW14] (see Theorem 15) but it does not seem to directly follow from them.

\footnotetext{
${ }^{5}$ We replace a linear function with its square to keep the sets homogeneous of degree 2.
} 
AMIR SHPILKA

\subsection{Proof Idea}

The basic tool in proving Theorems 7 and 8 is the following result that characterizes the different cases when a quadratic polynomial is in the radical of the ideal generated by two other quadratics, i.e., that is vanishes when the two quadratic polynomials vanish.

Theorem 10. Let $Q, Q_{1}, Q_{2}$ be such that whenever $Q_{1}$ and $Q_{2}$ vanish then also $Q$ vanishes. Then one of the following cases hold:

1. $Q$ is in the linear span of $Q_{1}, Q_{2}$

2. There exists a non trivial linear combination of the form $\alpha Q_{1}+\beta Q_{2}=\ell^{2}$ where $\ell$ is a linear function

3. There exist two linear functions $\ell_{1}$ and $\ell_{2}$ such that when setting $\ell_{1}=\ell_{2}=0$ we get that $Q, Q_{1}$ and $Q_{2}$ vanish.

The theorem guarantees that unless $Q$ is in the linear span of $Q_{1}$ and $Q_{2}$ then $Q_{1}$ and $Q_{2}$ must satisfy a very strong property, namely, they must span a square of a linear function or they have a very low rank (as quadratic polynomials). The proof of this theorem is based on analyzing the resultant of $Q_{1}$ and $Q_{2}$ with respect to some variable. We now explain how this theorem can be used to prove Theorem 7.

Consider a set of polynomials $\mathcal{T}=\left\{Q_{i}\right\}$ satisfying the condition of Theorem 7. If for every $Q \in \mathcal{T}$ for at least, say, $(1 / 100) \cdot|\mathcal{T}|$ of the polynomials $Q_{i} \in \mathcal{T}$ there is a another polynomial in $\operatorname{span}\left(Q, Q_{i}\right)$ then the claim follows by the robust version of the Sylvester-Gallai theorem proved in [BDWY13, DSW14] (Theorem 15). So let us assume this is not the case. And in fact, let us assume that there are two polynomials $Q_{1}, Q_{2} \in \mathcal{T}$ for which this does not hold. This means that at least 0.98 fraction of the polynomials in $\mathcal{T}$ satisfy Case 2 or Case 3 of Theorem 10 with $Q_{1}$ and $Q_{2}$. This gives very strong restriction on the structure of these $0.98 \cdot|\mathcal{T}|$ polynomials.

To use this structure we first show that the polynomials satisfying Case 2 of Theorem 10 with both $Q_{1}$ and $Q_{2}$ also span a low dimensional space (Claim 38). The intuition is that every such polynomial can be represented as both $\alpha Q_{1}+\ell_{1}^{2}$ and as $\beta Q_{2}+\ell_{2}^{2}$. This gives rise to many different equations involving $Q_{1}$ and $Q_{2}$. Analyzing those equations we show that all those $\ell_{i}$ span a low dimensional space.

The remaining polynomials must satisfy Case 3 of Theorem 10 with either $Q_{1}$ or $Q_{2}$. We then show (Claim 44) that, under the conditions of Theorem 7, all the polynomials that satisfy Case 3 of Theorem 10 with, say, $Q_{1}$ span a low dimensional space. The intuition is that if we map the linear functions in some "minimal" representation of $Q_{1}$ to a new variable $z$, then all these polynomials will be mapped to quadratics of the form $z \cdot \ell_{i}$. We then show that these $\ell_{i}$ 's satisfy the usual Sylvester-Gallai condition and hence get a bound on their span.

The proof outline of Theorem 8 involves more cases, but it is still similar in spirit and is based on studying the case where our three sets do not satisfy the robust version of the Edelstein-Kelly theorem (Theorem 9).

To prove Theorem 9 we would like to reduce to the robust version of the Sylvester-Gallai theorem proved in [BDWY13, DSW14]. For example, if all our sets are of the same size then their union forms a $\delta / 3-S G$ configuration (see subsection 2.2) and we can conclude using the result of [BDWY13, DSW14]. Thus, the main issue is what to do when the sets are of very different sizes. When the largest set has size 
polynomial in the size of the smallest set then we prove that by sampling a random subset of appropriate size from the largest set and taking its union with the two other sets we again get a $\delta / 6-S G$ configuration. This implies that the second largest and smallest sets live in an $O(1)$-dimensional space and hence all the sets span an $O(1)$-dimensional space. The proof of the case where the largest set is much larger than the smaller set is different and is based on a completely different covering argument.

\subsection{Organization}

The paper is organized as follows. section 2 contains basic facts regarding the resultant and some other basic tools and notation, including the robust version of the Sylvester-Gallai theorem of [BDWY13, DSW14]. In section 3 we define the notion of a $\delta$-EK configuration and prove Theorem 9. section 4 contains the proof of our structure theorem (Theorem 10). In section 5 we give the proof of Theorem 7 and in section 6 we prove Theorem 8. Finally in section 7 we discuss further directions and open problems.

\section{Preliminaries}

In this section we explain our notation, give some basic facts from algebra that will be useful in our proofs and state a robust version of the Sylvester-Gallai theorem.

We will mostly use the following notation. Greek letters $\alpha, \beta, \ldots$ denote scalars from the field. Uncapitalized letters $a, b, c, \ldots$ denote linear functions and $x, y, z$ denote variables (which are also linear functions). We denote $\mathbf{x}=\left(x_{1}, \ldots, x_{n}\right)$. Capital letters such as $A, Q, F$ denote quadratic polynomials whereas $V, U, W$ denote linear spaces. Calligraphic letters $\mathcal{J}, \mathcal{J}, \mathcal{F}, \mathcal{Q}, \mathcal{T}$ denote sets. For a positive integer $n$ we denote $[n]=\{1,2, \ldots, n\}$.

We will also need on the following version of Chernoff bound. See e.g. Theorem 4.5 in [MU05].

Theorem 11 (Chernoff bound). Suppose $X_{1}, \ldots, X_{n}$ are independent indicator random variables. Let $\mu=E\left[X_{i}\right]$ be the expectation of $X_{i}$. Then,

$$
\operatorname{Pr}\left[\sum_{i=1}^{n} X_{i}<\frac{1}{2} n \mu\right]<\exp \left(-\frac{1}{8} n \mu\right) .
$$

\subsection{Facts from algebra}

A notation that will convenient to use is that of a radical ideal. In this work we only consider the ring of polynomials $\mathbb{C}[\mathbf{x}]$. An ideal $I \subseteq \mathbb{C}[\mathbf{x}]$ is an abelian subgroup that is closed under multiplication by ring elements. We will denote with $\left(Q_{1}, Q_{2}\right)$ the ideal generated by two polynomials $Q_{1}$ and $Q_{2}$. I.e. $\left(Q_{1}, Q_{2}\right)=Q_{1} \cdot \mathbb{C}[\mathbf{x}]+Q_{2} \cdot \mathbb{C}[\mathbf{x}]$. The radical of an ideal $I$, denoted $\sqrt{I}$, is the set of all ring elements $f$ satisfying that for some natural number $m, f^{m} \in I$. Hilbert's Nullstellensatz implies that if a polynomial $Q$ vanishes whenever $Q_{1}$ and $Q_{2}$ vanish then $Q \in \sqrt{\left(Q_{1}, Q_{2}\right)}$ (see e.g. [CLO07]). We shall often use the notation $Q \in \sqrt{\left(Q_{1}, Q_{2}\right)}$ to denote this vanishing condition.

A tool that will play an important role in the proof of Theorem 10 is the resultant of two polynomials. As we only consider quadratic polynomials in this paper we restrict our attention to resultants of such 


\section{AMIR SHPILKA}

polynomials. Let $F, G \in \mathbb{C}[\mathbf{x}]$ be quadratic polynomials. View $F, G$ as polynomials in $x_{1}$ over $\mathbb{C}\left(x_{2}, \ldots, x_{n}\right)$. I.e.

$$
F=\alpha x_{1}^{2}+a x_{1}+F_{0} \quad \text { and } G=\beta x_{1}^{2}+b x_{1}+G_{0} .
$$

Then, the resultant of $F$ and $G$ with respect to $x_{1}$ is the determinant of their Sylvester matrix

$$
\operatorname{Res}_{x_{1}}(F, G):=\left|\left[\begin{array}{cccc}
F_{0} & 0 & G_{0} & 0 \\
a & F_{0} & b & G_{0} \\
\alpha & a & \beta & b \\
0 & \alpha & 0 & \beta
\end{array}\right]\right| .
$$

A useful fact is that if the resultant of $F$ and $G$ vanishes then they share a common factor.

Theorem 12 (See e.g. Proposition 8 in $\S 5$ of Chapter 3 in [CLO07]). Given $F, G \in \mathbb{F}[x]$ of positive degree, the resultant $\operatorname{Res}_{x}(F, G)$ is an integer polynomial in the coefficients of $F, G$. Furthermore, $F$ and $G$ have a common factor in $\mathbb{F}[x]$ if and only if $\operatorname{Res}_{x}(F, G)=0$.

Finally, we shall define the rank of a quadratic polynomial as follows.

Definition 13. For a quadratic polynomial we denote with $\operatorname{rank}_{s}(Q)$ the minimal $r$ such that there are $2 r$ linear functions $\left\{\ell_{i}\right\}_{i=1}^{2 r}$ satisfying $Q=\sum_{i=1}^{r} \ell_{2 i} \cdot \ell_{2 i-1}$. We call such a representation a minimal representation of $Q$.

This is a slightly different definition than the usual way one defines rank of quadratic forms, but it is more suitable for our needs. We note that a quadratic $Q$ is irreducible if and only if $\operatorname{rank}_{s}(Q)>1$. The next claim shows that a minimal representation is unique in the sense that the space spanned by the linear functions in it is unique.

Claim 14. Let $Q$ be an irreducible quadratic polynomial with $\operatorname{rank}_{s}(Q)=r$. Let $Q=\sum_{i=1}^{r} a_{2 i-1} \cdot a_{2 i}$ and $Q=\sum_{i=1}^{r} b_{2 i-1} \cdot b_{2 i}$ be two different minimal representations of $Q$. Then $\operatorname{span}\left\{a_{i}\right\}=\operatorname{span}\left\{b_{i}\right\}$.

Proof. Note that if the statement does not hold then, w.l.o.g., $a_{1}$ is not contained in the span of the $b_{i}$ 's. This means that when setting $a_{1}=0$ the $b_{i}$ 's are not affected on the one hand, thus $Q$ remains the same function of the $b_{i}$ 's, and in particular $\operatorname{rank}_{s}\left(\left.Q\right|_{a_{1}=0}\right)=r$, but on the other hand $\operatorname{rank}_{s}\left(\left.Q\right|_{a_{1}=0}\right)=r-1$ (when considering its representation with the $a_{i}$ 's), in contradiction.

\subsection{Robust Sylvester-Gallai theorem}

We will need the following theorem of Dvir et al. [DSW14] that improves on an earlier work of Barak et al. [BDWY13].

We say that the points $v_{1}, \ldots, v_{m}$ in $\mathbb{C}^{d}$ form a $\delta$-SG configuration if for every $i \in[m]$ there exists at least $\delta m$ values of $j \in[m]$ such that the line through $v_{i}, v_{j}$ contains a third point in the set.

Theorem 15 (Theorem 1.9 of [DSW14]). If $v_{1}, \ldots, v_{m} \in \mathbb{C}^{d}$ is a $\delta$-SG configuration then $\operatorname{dim}\left(\operatorname{span}\left\{v_{1}, \ldots, v_{m}\right\}\right) \leq 12 / \delta$.

An easy consequence of the theorem is the following. 
Corollary 16. Let $0<\delta<1$. Assume $v_{0}, v_{1}, \ldots, v_{m} \in \mathbb{C}^{d}$ are such that for every $i \in[m]$ there exists at least $\delta m$ values of $j \in[m]$ such that the line through $v_{i}, v_{j}$ contains a third point in the set (i.e. the condition holds for all the points except, possibly, $\left.v_{0}\right)$. Then $\operatorname{dim} v_{0}, v_{1}, \ldots, v_{m}<50 / \delta$.

Proof. The only way that $v_{0}, v_{1}, \ldots, v_{m}$ fail to be a $\delta$-SG configuration is if $v_{0}$ does not satisfy the condition. By considering all pairs $\left(v_{i}, v_{j}\right)$ that lie on a line with $v_{0}$ we conclude that either $v_{0}, v_{1}, \ldots, v_{m}$ is a $\frac{\delta}{2}$-SG configuration or $v_{1}, \ldots, v_{m}$ is. In any case, by Theorem 15 , we get that $\operatorname{dim} v_{1}, \ldots, v_{m} \leq 48 / \delta$ and the total dimension is at most $50 / \delta$.

Remark 17. In our application we will have that the span of two points contains a third point. This does not change the theorems much as by picking a random subspace $H$, of codimension 1 , and replacing each point $p$ with $H \cap \operatorname{span}\{p\}$ we get that $p_{3} \in \operatorname{span}\left\{p_{1}, p_{2}\right\}$ iff $H \cap \operatorname{span}\left\{p_{3}\right\}$ is on the line passing through $H \cap \operatorname{span}\left\{p_{1}\right\}$ and $H \cap \operatorname{span}\left\{p_{2}\right\}$.

\section{Robust Edelstein-Kelly theorems}

In this section we prove Theorem 9 as well as some extensions of it, which give robust versions of the following theorem of Edelstein and Kelly [EK66].

Theorem 18 (Theorem 3 of [EK66]). Let $\mathcal{T}_{i}$, for $i \in[3]$, be disjoint finite subsets of $\mathbb{C}^{n}$ such that for every $i \neq j$ and any two points $p_{1} \in \mathcal{T}_{i}$ and $p_{2} \in \mathcal{T}_{j}$ there exists a point $p_{3}$ in the third set that is on the line passing through $p_{1}$ and $p_{2}$. Then, any such $\mathcal{T}_{i}$ satisfy that $\operatorname{dim}\left(\operatorname{span}\left\{\cup_{i} \mathcal{T}_{i}\right\}\right) \leq 3$.

We would be interested in the case where the requirement in the theorem holds with some positive probability. We say that the sets $\mathcal{T}_{1}, \mathcal{T}_{2}, \mathcal{T}_{3} \subset \mathbb{C}^{n}$ form a $\delta$-EK configuration if for every $i \in[3]$ and $p \in \mathcal{T}_{i}$, for every $j \in[3] \backslash\{i\}$ at least $\delta$ fraction of the points $p_{j} \in \mathcal{T}_{j}$ satisfy that $p$ and $p_{j}$ span some point in the third set. ${ }^{6}$ To ease the reading we state again Theorem 9.

Theorem (Theorem 9). Let $0<\delta \leq 1$ be any constant. Let $\mathcal{T}_{1}, \mathcal{T}_{2}, \mathcal{T}_{3} \subset \mathbb{C}^{n}$ be disjoint finite subsets that form a $\delta$-EK configuration. Then $\operatorname{dim}\left(\operatorname{span}\left\{\cup_{i} \mathcal{T}_{i}\right\}\right) \leq O\left(1 / \delta^{3}\right)$.

Proof of Theorem 9. Denote $\left|\mathcal{T}_{i}\right|=m_{i}$. Assume w.l.o.g. that $\left|\mathcal{T}_{1}\right| \geq\left|\mathcal{T}_{2}\right| \geq\left|\mathcal{T}_{3}\right|$. The proof distinguishes two cases. The first is when $\left|\mathcal{T}_{3}\right|$ is not too small and the second case is when it is much smaller than the largest set.

1. Case $m_{3}>m_{1}^{1 / 3}$ :

Let $\mathcal{T}_{1}^{\prime} \subset \mathcal{T}_{1}$ be a random subset, where each element is samples with probability $m_{2} / m_{1}=\left|\mathcal{T}_{2}\right| / \mid \mathcal{T}_{1}$. By the Chernoff bound (Theorem 11) we get that, w.h.p., the size of the set is at most, say, $2 m_{2}$. Further, the Chernoff bound also implies that for every $p \in \mathcal{T}_{2}$ there are at least $(\delta / 2) \cdot m_{2}$ points in $\mathcal{T}_{1}^{\prime}$ that together with $p$ span a point in $\mathcal{T}_{3}$. Similarly, for every $p \in \mathcal{T}_{3}$ there are at least $(\delta / 2) \cdot m_{2}$ points in $\mathcal{T}_{1}^{\prime}$ that together with $p$ span a point in $\mathcal{T}_{2}$. Clearly, we also have that for every point

\footnotetext{
${ }^{6}$ Note that here we use the notion of span rather than a line passing through points. However, as noted in Remark 17 , this does not make any real difference.
} 


\section{AMIR SHPILKA}

$p \in \mathcal{T}_{1}^{\prime}$ there are $\delta m_{2}$ points in $\mathcal{T}_{2}$ that together with $p$ span a point in $\mathcal{T}_{3}$. Thus, the set $\mathcal{T}_{1}^{\prime} \cup \mathcal{T}_{2} \cup \mathcal{T}_{3}$ is a $(\delta / 8)$-SG configuration and hence has dimension $O(1 / \delta)$ by Theorem 15 .

Let $V$ be a subspace of dimension $O(1 / \delta)$ containing all these points. Note that in particular, $\mathcal{T}_{2}, \mathcal{T}_{3} \subset V$. As every point $p \in \mathcal{T}_{1}$ is a linear combination of points in $\mathcal{T}_{2} \cup \mathcal{T}_{3}$ it follows that the whole set has dimension $O(1 / \delta)$.

1. Case $m_{3} \leq m_{1}^{1 / 3}$ :

In this case we may not be able to use the sampling approach from earlier as $m_{2}$ can be too small and the Chernoff argument from above will not hold.

We say that a point $p_{1} \in \mathcal{T}_{1}$ is a neighbor of a point $p \in \mathcal{T}_{2} \cup \mathcal{T}_{3}$ if the space spaned by $p$ and $p_{1}$ intersects the third set. Denote with $\Gamma_{1}(p)$ the neighborhood of a point $p \in \mathcal{T}_{2} \cup \mathcal{T}_{3}$ in $\mathcal{T}_{1}$.

Every two points $p \in \mathcal{T}_{2}$ and $q \in \mathcal{T}_{3}$ define a two-dimensional space that we denote $V(p, q)=$ $\operatorname{span}\{p, q\}$.

Fix $p \in \mathcal{T}_{2}$ and consider those spaces $V(p, q)$ that contain points from $\mathcal{T}_{1}$. Clearly there are at most $\left|\mathcal{T}_{3}\right|$ such spaces. Any two different subspaces $V\left(p, q_{1}\right)$ and $V\left(p, q_{2}\right)$ have intersection of dimension 1 (it is $\operatorname{span}\{p\}$ ) and by the assumption in the theorem the union $\cup_{q \in \mathcal{T}_{3}} V(p, q)$ covers at least $\delta m_{1}$ points of $\mathcal{T}_{1}$. Indeed, $\delta m_{1}$ points $q_{1} \in \mathcal{T}_{1}$ span a point in $\mathcal{T}_{3}$ together with $p$. As our points are pairwise independent, it is not hard to see that if $q_{3} \in \operatorname{span}\left\{p, q_{1}\right\}$ then $q_{1} \in \operatorname{span}\left\{p, q_{3}\right\}=V\left(p, q_{3}\right)$

For each subspace $V(p, q)$ consider the set $V(p, q)_{1}=V(p, q) \cap \mathcal{T}_{1}$.

Claim 19. Any two such spaces $V\left(p, q_{1}\right)$ and $V\left(p, q_{2}\right)$ satisfy that either $V\left(p, q_{1}\right)_{1}=V\left(p, q_{2}\right)_{1}$ or $V\left(p, q_{1}\right)_{1} \cap V\left(p, q_{2}\right)_{1}=\emptyset$.

Proof. If there was a point $p^{\prime} \in V\left(p, q_{1}\right)_{1} \cap V\left(p, q_{1}\right)_{1}$ then both $V\left(p, q_{1}\right)$ and $V\left(p, q_{2}\right)$ would contain $p, p^{\prime}$ and as $p$ and $p^{\prime}$ are linearly independent (since they belong to $\mathcal{T}_{i}$ 's they are not the same point) that would make $V\left(p, q_{1}\right)=V\left(p, q_{2}\right)$. In particular we get $V\left(p, q_{1}\right)_{1}=V\left(p, q_{2}\right)_{1}$.

As conclusion we see that at most $O\left(1 / \delta^{2}\right)$ different spaces $\{V(p, q)\}_{q}$ have intersection at least $\delta^{2} / 100 \cdot m_{1}$ with $\mathcal{T}_{1}$. Let $\mathcal{J}$ contain $p$ and a point from each of the sets $\left\{V(p, q)_{1}\right\}$ that have size at least $\delta^{2} / 100 \cdot m_{1}$. Clearly $|\mathcal{J}| \leq O\left(1 / \delta^{2}\right)$. We now repeat the following process. As long as $\mathcal{T}_{2} \not \subset \operatorname{span}\{\mathcal{J}\}$ we pick a point $p^{\prime} \in \mathcal{T}_{2} \backslash \operatorname{span}\{\mathcal{J}\}$. We add $p^{\prime}$ to $\mathcal{J}$ along with a point from each large set $V\left(p^{\prime}, q\right)_{1}$, i.e. subsets satisfying $\left|V\left(p^{\prime}, q\right)_{1}\right| \geq \delta^{2} / 100 \cdot m_{1}$, and repeat.

We next show that this process must terminate after $O(1 / \delta)$ steps and that at the end $|\mathcal{J}|=O\left(1 / \delta^{3}\right)$. To show that the process terminates quickly we prove that if $p_{k} \in \mathcal{T}_{2}$ is the point that was picked at the $k$ 'th step then $\left|\Gamma_{1}\left(p_{k}\right) \backslash \cup_{i \in[k-1]} \Gamma_{1}\left(p_{i}\right)\right| \geq(\delta / 2) m_{1}$. Thus, every step covers at least $\delta / 2$ fraction of new points in $\mathcal{T}_{1}$ and thus the process must end after at most $O(1 / \delta)$ steps.

Claim 20. Let $p_{i} \in \mathcal{T}_{2}$, for $i \in[k-1]$ be the point chosen at the ith step. If the intersection of $V\left(p_{k}, q\right)$ with $V\left(p_{i}, q^{\prime}\right){ }_{1}$, for any $q, q^{\prime} \in \mathcal{T}_{3}$, has size larger than 1 then $V\left(p_{k}, q\right)=V\left(p_{i}, q^{\prime}\right)$ (and in particular, $\left.V\left(p_{k}, q\right)_{1}=V\left(p_{i}, q^{\prime}\right)_{1}\right)$ and $\left|V\left(p_{k}, q\right)_{1}\right| \leq \delta^{2} / 100 \cdot m_{1}$.

Moreover, if there is another pair $\left(q^{\prime \prime}, q^{\prime \prime \prime}\right) \in \mathcal{T}_{3}^{2}$ satisfying $\left|V\left(p_{k}, q^{\prime \prime}\right)_{1} \cap V\left(p_{i}, q^{\prime \prime \prime}\right)_{1}\right|>1$ then it must be the case that $V\left(p_{i}, q^{\prime}\right)=V\left(p_{i}, q^{\prime \prime \prime}\right)$. 
Proof. If the intersection of $V\left(p_{k}, q\right)_{1}$ with $V\left(p_{i}, q^{\prime}\right)_{1}$ has size at least 2 then by an argument similar to the proof of Claim 19 we would get that $V\left(p_{k}, q\right)=V\left(p_{i}, q^{\prime}\right)$. To see that in this case the size of $V\left(p_{i}, q^{\prime}\right)_{1}$ is not too large we note that by our process, if $\left|V\left(p_{i}, q^{\prime}\right)_{1}\right| \geq \delta^{2} / 100 \cdot m_{1}$ then $\mathcal{J}$ contains at least two points from $V\left(p_{i}, q^{\prime}\right)_{1}$. Hence, $p_{k} \in V\left(p_{i}, q^{\prime}\right) \subset \operatorname{span}\{\mathcal{J}\}$ in contradiction to the choice of $p_{k}$.

To prove the moreover part we note that in the case of large intersection, since $V\left(p_{k}, q\right)=V\left(p_{i}, q^{\prime}\right)$, we have that $p_{k}, p_{i} \in V\left(p_{i}, q^{\prime}\right)$. If there was another pair $\left(q^{\prime \prime}, q^{\prime \prime \prime}\right)$ so that $\left|V\left(p_{k}, q^{\prime \prime}\right)_{1} \cap V\left(p_{i}, q^{\prime \prime \prime}\right)_{1}\right|>$ 1 then we would similarly get that $p_{k}, p_{i} \in V\left(p_{i}, q^{\prime \prime \prime}\right)$. By pairwise linear independence of the points in our sets this implies that $V\left(p_{i}, q^{\prime}\right)=V\left(p_{i}, q^{\prime \prime \prime}\right)$.

Corollary 21. Let $i \in[k-1]$ then

$$
\left|\Gamma_{1}\left(p_{k}\right) \cap \Gamma_{1}\left(p_{i}\right)\right| \leq \delta^{2} / 100 \cdot m_{1}+m_{3}^{2} .
$$

Proof. The proof follows immediately from Claim 20. Indeed, the claim assures that there is at most one subspace $V\left(p_{k}, q\right)$ that has intersection of size larger than 1 with any $V\left(p_{i}, q^{\prime}\right)_{1}$ (and that there is at most one such subspace $\left.V\left(p_{i}, q^{\prime}\right)\right)$ and that whenever the intersection size is larger than 1 it is upper bounded by $\delta^{2} / 100 \cdot m_{1}$. As there are at most $m_{3}^{2}$ pairs $\left(q, q^{\prime}\right) \in \mathcal{T}_{3}^{2}$ the claim follows.

The corollary implies that

$$
\left|\Gamma_{1}\left(p_{k}\right) \cap\left(\cup_{i \in[k-1]} \Gamma_{1}\left(p_{i}\right)\right)\right| \leq k\left(\left(\delta^{2} / 100\right) m_{1}+m_{3}^{2}\right)<(\delta / 2) \cdot m_{1},
$$

where the last inequality holds for, say, $k<10 / \delta .^{7}$ As $\left|\Gamma_{1}\left(p_{k}\right)\right| \geq \delta \cdot m_{1}$, for each $k$, it follows that after $k<10 / \delta$ steps

$$
\left|\cup_{i \in[k]} \Gamma_{1}\left(p_{i}\right)\right|>k(\delta / 2) m_{1} .
$$

In particular, the process must end after at most $2 / \delta$ steps.

As each steps adds to $\mathcal{J}$ at most $O\left(1 / \delta^{2}\right)$ vectors, at the end we have that $|\mathcal{J}|=O\left(1 / \delta^{3}\right)$ and every $p \in \mathcal{T}_{2}$ is in the span of $\mathcal{J}$.

Now that we have proved that $\mathcal{T}_{2}$ has small dimension we conclude as follows. We find a maximal subset of $\mathcal{T}_{3}$ whose neighborhood inside $\mathcal{T}_{1}$ are disjoint. As each neighborhood has size at least $\delta \cdot m_{1}$ it follows there the size of the subset is at most $O(1 / \delta)$. We add those $O(1 / \delta)$ points to J and let $V=\operatorname{span}\{\mathcal{J}\}$. Clearly $\operatorname{dim}(V)=O\left(1 / \delta^{3}\right)$.

Claim 22. $\cup_{i} \mathcal{T}_{i} \subset V$.

Proof. We first note that if $p \in \mathcal{T}_{1}$ is in the neighborhood of some $p^{\prime} \in \mathcal{J} \cap \mathcal{T}_{3}$ then $p \in V$. Indeed, the subspace spanned by $p^{\prime}$ and $p$ intersects $\mathcal{T}_{2}$. I.e. there is $q \in \mathcal{T}_{2}$ that is equal to $\alpha p+\beta p^{\prime}$, where from pairwise independence both $\alpha \neq 0$ and $\beta \neq 0$. As both $p^{\prime} \in V$ and $\mathcal{T}_{2} \subset V$ we get that also $p \in V$.

\footnotetext{
${ }^{7}$ It is here that we use the fact that we are in the case $m_{3} \leq m_{1}^{1 / 3}$.
} 


\section{AMIR SHPILKA}

We now have that the neighborhood of every $p \in \mathcal{T}_{3} \backslash \mathcal{J}$ intersects the neighborhood of some $p^{\prime} \in \mathcal{J} \cap \mathcal{T}_{3}$. Thus, there is some point $q \in \mathcal{T}_{1}$ that is in $V$ (by the argument above as it is a neighbor of $p^{\prime}$ ) and is also a neighbor of $p$. It follows that also $p \in V$ as the subspace spanned by $q$ and $p$ contains some point in $\mathcal{T}_{2}$ and both $\{q\}, \mathcal{T}_{2} \subset V$ (and we use pairwise independence again). Hence all the points in $\mathcal{T}_{3}$ are in $V$. As $\mathcal{T}_{2} \cup \mathcal{T}_{3} \subset V$ it follows that also $\mathcal{T}_{1} \subset V$.

This concludes the proof of the case $m_{3} \leq m_{1}^{1 / 3}$.

Remark 23. The bound $O\left(1 / \delta^{3}\right)$ is probably not tight and we believe that the correct bound should be $O(1 / \delta)$ but we did not try to get tight bounds here. The theorem also seems similar in spirit to the results in [BDWY13, DSW14] but as far as we can tell it is not a direct corollary of any of the results there.

Remark 24. While Theorem 9 speaks about lines through points, a similar conclusion holds when we replace the condition that $p_{3}$ lies on the line through $p_{1}$ and $p_{2}$ with the condition $p_{3} \in \operatorname{span}\left\{p_{1}, p_{2}\right\}$.

Similar to Corollary 16 we have the following variant of Theorem 9.

Theorem 25. Let $0<\delta \leq 1$ be any constant. Let $\mathcal{T}_{1}, \mathcal{T}_{2}, \mathcal{T}_{3} \subset \mathbb{C}^{n}$ be disjoint finite subsets. Assume that with the exception of at most c elements from $\cup_{i=1}^{3} \mathcal{T}_{i}$ all other elements in $\cup_{i=1}^{3} \mathcal{T}_{i}$ satisfy the $\delta$-EK property. Then $\operatorname{dim}\left(\operatorname{span}\left\{\cup_{i} \mathcal{T}_{i}\right\}\right) \leq O_{c}\left(1 / \delta^{3}\right)$.

Sketch. The proof is similar to the proof of Theorem 9 so we just explain how to modify it.

1. Case $m_{3}>m_{1}^{1 / 3}$ : Here too we repeat the sampling argument and note, similar to Corollary 16 that the sampled set give rise to an $\Omega\left(\delta / 2^{c}\right)$-SG configuration. Adding the $c$ " "bad" elements to the subspace $V$ gives a subspace of dimension $O_{c}(1 / \delta)$ spanning $\mathcal{T}_{2} \cup \mathcal{T}_{3}$. The rest of the proof is the same.

2. Case $m_{3} \geq m_{1}^{1 / 3}$ : We repeat the covering argument only now we initiate $\mathcal{J}$ with the $c$ " "bad" elements. It is not hard to see that the rest of the proof gives the desired result.

For the proof of Theorem 8 we would actually need the following extension of the theorem. The extension speaks of a situation where some linear combinations fall into a subspace $W$ and not just to one of the sets.

Theorem 26. Let $0<\delta \leq 1$ be any constant. Let $W \subset \mathbb{C}^{n}$ be an $r$-dimensional space and let $W_{i} \subset W$, for $i \in[3]$, be finite subsets of $W$. Let $\mathcal{K}_{1}, \mathcal{K}_{2}, \mathcal{K}_{3} \subset \mathbb{C}^{n} \backslash W$ be finite subsets. Let $\mathcal{T}_{i}=\mathcal{K}_{i} \cup W_{i}$. Assume that no two vectors in $\cup_{i} \mathcal{T}_{i}$ are linearly dependent.

Assume that with the exception of at most c elements from $\cup_{i=1}^{3} \mathcal{K}_{i}$ all other elements satisfy the following relaxed EK-property: If $p \in \mathcal{K}_{i}$ is not one of the c exceptional points then for every $j \in[3] \backslash\{i\}$, for at least $\delta$ fraction of the points $q \in \mathcal{T}_{j}$ the span of $p$ and $q$ contains a point in $\mathcal{T}_{k}$, for the third index $k$. Then, there exists a linear subspace $V$ of dimension $\operatorname{dim}(V)=O_{c}\left(1 / \delta^{3}\right)$ such that $\operatorname{span}\left\{\cup_{i} \mathcal{T}_{i}\right\} \subseteq W+V$. In particular, $\operatorname{dim}\left(\operatorname{span}\left\{\cup_{i} \mathcal{T}_{i}\right\}\right) \leq O_{c}\left(r+1 / \delta^{3}\right)$. 
Note that the theorem assumes nothing about the relation between the size of $W_{i}$ and $\mathcal{K}_{i}$. Furthermore, it only asks that points in $\mathcal{K}_{i}$ satisfy the spanning property with points from $\mathcal{T}_{j}=\mathcal{K}_{j} \cup W_{j}$ and that the spanned point can belong to $\mathcal{T}_{k}=\mathcal{K}_{k} \cup W_{k}$ and not just to $\mathcal{K}_{k}$.

Proof. As in the proof of Theorem 9 we denote the neighborhood of an element $p \in \cup_{i} \mathcal{K}_{i}$ in $\mathcal{T}_{j}$ with $\Gamma_{j}(p)$. I.e., $q \in \mathcal{T}_{j}$ belongs to $\Gamma_{j}(p)$, for $p \in \mathcal{K}_{i}(i \neq j)$, if $p$ and $q$ span a point in $\mathcal{T}_{k}$ where $k \notin\{i, j\}$. Assume w.l.o.g. that $\left|\mathcal{K}_{1}\right| \geq\left|\mathcal{K}_{2}\right| \geq\left|\mathcal{K}_{3}\right|$. As in the proof of Theorem 9 we distinguish two cases.

1. Case $\left|\mathcal{K}_{3}\right|>\left|\mathcal{K}_{1}\right|^{1 / 3}$ :

Our first step is to project the space $W$ to a random one-dimensional space $W_{0}=\operatorname{span}\left\{w_{0}\right\} \subset W$. We do so by projecting $\mathbb{C}^{n}$ to $\mathbb{C}^{n-r+1}$ in a way that the kernel of the projection is in $W$. Note that if we pick $w_{0} \in W$ at random (by, say, picking its coefficients uniformly from $[0,1]^{r}$ ) then any two vectors from $\cup_{i} \mathcal{K}_{i}$ remain linearly independent with probability 1 . We also note that this projection does not affect linear dependencies.

We abuse notation and use $\mathcal{K}_{i}$ to denote the set $\mathcal{K}_{i}$ after the projection. In contrast to the $\mathcal{K}_{i}$ 's, all elements from $W$ now become linearly dependent. Thus, if $W_{i}$ was not empty then we now replace it with the single vector $w_{0}$.

We now proceed as in the proof of Theorem 9 and sample a random subset $\mathcal{K}_{1}^{\prime} \subseteq \mathcal{K}_{1}$ of size roughly $\left|\mathcal{K}_{2}\right|$ (i.e. each element of $\mathcal{K}_{1}$ is added to the set with probability $\left.\left|\mathcal{K}_{2}\right| /\left|\mathcal{K}_{1}\right|\right)$. We would like to show that the new sets satisfy the conditions of Theorem 25 with parameter $\delta / 4$ and $c+1$ bad polynomials.

Claim 27. $\mathcal{K}_{1}^{\prime} \cup \mathcal{K}_{2} \cup \mathcal{K}_{3} \cup\left\{w_{0}\right\}$ satisfy the conditions of Theorem 25 with parameter $\delta / 4$ and at most $c+1$ bad polynomials..

Proof. Consider an element $p \in \mathcal{K}_{1}^{\prime}$ that is not exceptional. Then, before the projection to $W_{0}$, there were $\delta\left|\mathcal{T}_{2}\right|$ elements of $\mathcal{T}_{2}$ that each, together with $p$, spanned a point in $\mathcal{K}_{3} \cup W$. I.e., $\left|\Gamma_{2}(p)\right| \geq \delta\left|\mathcal{T}_{2}\right|$. Observe that some of the elements from $\Gamma_{2}(p)$ may have been projected to a multiple of $w_{0}$. We wish to show that in any case there are many points in $\mathcal{K}_{1}^{\prime} \cup \mathcal{K}_{2} \cup \mathcal{K}_{3} \cup\left\{w_{0}\right\}$ that together with $p$ span a third point in the set. We consider two cases.

(a) $\left|\Gamma_{2}(p) \cap W\right| \leq(\delta / 2)\left|\mathcal{T}_{2}\right|$ : In this case

$$
\left|\Gamma_{2}(p) \cap \mathcal{K}_{2}\right|=\left|\Gamma_{2}(p)\right|-\left|\Gamma_{2}(p) \cap W\right| \geq \delta\left|\mathcal{T}_{2}\right|-(\delta / 2)\left|\mathcal{T}_{2}\right|=(\delta / 2)\left|\mathcal{T}_{2}\right| \geq(\delta / 2)\left|\mathcal{K}_{2}\right| .
$$

(b) $\left|\Gamma_{2}(p) \cap W\right|>(\delta / 2)\left|\mathcal{T}_{2}\right|$ : Observe that for any $q \in \Gamma_{2}(p) \cap W$ the space spanned by $p$ and $q$ must contain a point in $\mathcal{K}_{3}$ as otherwise we would get that $p \in W$ as well, contradicting the assumption that $\mathcal{K}_{1} \subset \mathbb{C}^{n} \backslash W$. Furthermore, all the points in $\mathcal{K}_{3}$ that are obtained in this manner must be distinct. Indeed, if $p$ spans $q \in \mathcal{K}_{3}$ with $w_{1}, w_{2} \in W_{2}$ then, as $w_{1}$ and $w_{2}$ are linearly independent and so are $p$ and $q$, we get that $\operatorname{span}\{p, q\}=\operatorname{span}\left\{w_{1}, w_{2}\right\}$ and again it follows that $p \in W$. It therefore follows that $p$ spans a point in $W$ with at least

$$
(\delta / 2)\left|\mathcal{T}_{2}\right| \geq(\delta / 2)\left|\mathcal{K}_{2}\right|
$$

elements of $\mathcal{K}_{3}$. As any two points in $\cup_{i} \mathcal{K}_{i}$ remained linearly independent after the projection of $W$ to $W_{0}$, it follows that $p$ spans $w_{0}$ with at least $(\delta / 2)\left|\mathcal{K}_{2}\right|$ elements of $\mathcal{K}_{3}$. 


\section{AMIR SHPILKA}

Thus, in any case $p$ has at least $(\delta / 2) \cdot\left|\mathcal{K}_{2}\right|$ points in $\mathcal{K}_{2} \cup \mathcal{K}_{3}$ that together with it span a third point in $\mathcal{K}_{1}^{\prime} \cup \mathcal{K}_{2} \cup \mathcal{K}_{3} \cup\left\{w_{0}\right\}$.

A similar argument shows roughly the same result for $p \in \mathcal{K}_{2} \cup \mathcal{K}_{3}$, where now we also have to remember to use the Chernoff bound to claim that the fraction of neighbors it has in $\mathcal{K}_{1}^{\prime}$ is roughly the same as in $\mathcal{K}_{1}$, namely, at least $\delta / 2$ (similarly to the proof of Theorem 9).

We therefore have a set of size at most, say, $4\left|\mathcal{K}_{2}\right|+1$ that, with the possible exception of $c+1$ points (the original $c$ points and $w_{0}$ ), satisfy the condition of Theorem 25 with parameter $\delta / 4$ (we lose a factor of 2 in $\delta$ when sampling $\mathcal{K}_{1}^{\prime}$ and then another factor due to the projection to $W_{0}$ ).

We continue with the proof of Theorem 26. Claim 27 and Theorem 25 imply that the projected set $\mathcal{K}_{1}^{\prime} \cup \mathcal{K}_{2} \cup \mathcal{K}_{3} \cup W_{0}$ is contained in a subspace $V$ of dimension at most $O_{c}\left(1 / \delta^{3}\right)$. As we projected $W$ to span $w_{0} \subseteq W$, it follows that $\mathcal{K}_{1}^{\prime} \cup \mathcal{K}_{2} \cup \mathcal{K}_{3} \cup W \subset V+W$, and clearly $\operatorname{dim}(V+W)=O_{c}\left(r+1 / \delta^{3}\right)$. All that is left is to extend the bound to include $\mathcal{K}_{1}$ instead of $\mathcal{K}_{1}^{\prime}$ and this is done as in the proof of Theorem 9 without losing much in the dimension of $V$ (except a possible additive term of $c$ to $\operatorname{dim}(V)$ ). We thus get that $\mathcal{K}_{1} \cup \mathcal{K}_{2} \cup \mathcal{K}_{3} \cup W \subseteq V+W$. This of course implies that $\mathcal{T}_{1} \cup \mathcal{T}_{2} \cup \mathcal{T}_{3} \subseteq V+W$ and $\operatorname{dim}(V+W)=O_{c}\left(r+1 / \delta^{3}\right)$ as claimed.

2. Case $\left|\mathcal{K}_{3}\right| \leq\left|\mathcal{K}_{1}\right|^{1 / 3}$ :

The proof in this case is similar to the second case in the proof of Theorem 9.

Note that, since $\mathcal{K}_{1}$ is so large and every $p \in \mathcal{K}_{2}$ has at least $\delta\left|\mathcal{T}_{1}\right|$ neighbors in $\mathcal{T}_{1}$, we get that $p$ also has at least $(\delta / 2) \cdot\left|\mathcal{K}_{1}\right|$ neighbors in $\mathcal{K}_{1}$. Indeed, as before if a neighbor $q$ of $p$ is in $W_{1}$ then the third point spanned by $p$ and $q$ cannot be in $W_{3}$. Hence it must be in $\mathcal{K}_{3}$. Again it is easy to show that all the elements in $\mathcal{K}_{3}$ that are obtained in this way must be distinct and since the set $\mathcal{K}_{3}$ is too small the claim follows.

We now proceed as in the proof of Theorem 9. For $p \in \mathcal{K}_{2}$ and $q \in \mathcal{K}_{3}$ we define the two dimensional space $V(p, q)=\operatorname{span}\{p, q\}$ and denote $V(p, q)_{1}=V(p, q) \cap \mathcal{K}_{1}$.

Let $\mathcal{J}$ contain the $c$ exceptional points. Consider $p_{1} \in \mathcal{K}_{2}$ that is not in the span of the points in $\mathcal{J}$. Add $p_{1}$ to $\mathcal{J}$ as well as any $q \in \mathcal{K}_{3}$ so that $\left|V\left(p_{1}, q\right)_{1}\right|>\left((\delta / 2)^{2} / 100\right) \cdot\left|\mathcal{K}_{1}\right|$. Continue this process where at each step $i$ we pick $p_{i} \in \mathcal{K}_{2}$ that is not in the linear span of the vectors in $\mathcal{J}$. We continue doing so noting that at east step the number of vectors in $\mathcal{K}_{1}$ that is covered by the neighborhoods of the points $p_{i}$ that we picked grows by at least $(1 / 2) \cdot(\delta / 2) \cdot\left|\mathcal{K}_{1}\right|$ (the argument is the same as in the second case in the proof of Theorem 9). Hence, the process must terminate after $O(1 / \delta)$ steps at which stage $\mathcal{J}$ is of size $O_{c}\left(1 / \delta^{3}\right)$. As in the second case in the proof of Theorem 9 we conclude that $\mathcal{J}$ spans all points in $\mathcal{K}_{2}$.

We continue as in the proof of Theorem 9. We find a maximal subset of $\mathcal{K}_{3}$ whose neighborhood inside $\mathcal{T}_{1}$ are disjoint. As each neighborhood has size at least $\delta \cdot\left|\mathcal{T}_{1}\right|$ it follows there the size of the subset is at most $O(1 / \delta)$. We add those $O(1 / \delta)$ points to $\mathcal{J}$ and let $V=\operatorname{span}\{\mathcal{J}\}$. Clearly $\operatorname{dim}(V)=O_{c}\left(1 / \delta^{3}\right)$. As in the proof of Theorem 9 we have that $\mathcal{T}_{2} \subset V+W$.

Claim 28. $\cup_{i} \mathcal{T}_{i} \subset V+W$. 
Proof. As before, note that if $p \in \mathcal{T}_{1}$ is in the neighborhood of some $p^{\prime} \in \mathcal{J} \cap \mathcal{K}_{3}$ then $p \in V+W$. Indeed, the subspace spanned by $p^{\prime}$ and $p$ intersects $\mathcal{T}_{2}$. I.e. there is $q \in \mathcal{T}_{2}$ that is equal to $\alpha p+\beta p^{\prime}$, where from pairwise independence it follows that $\alpha \neq 0$ and $\beta \neq 0$. As $p^{\prime} \in V$ and $\mathcal{T}_{2} \subset V+W$ it also holds that $p \in V+W$.

We now have that the neighborhood of every $p \in \mathcal{K}_{3} \backslash \mathcal{J}$ intersects the neighborhood of some $p^{\prime} \in \mathcal{J} \cap \mathcal{K}_{3}$. Thus, there is some point $q \in \mathcal{T}_{1}$ that is in $V+W$ (by the argument above, as it is a neighbor of $p^{\prime}$ ) and is also a neighbor of $p$. It follows that also $p \in V+W$ as the subspace spanned by $q$ and $p$ contains some point in $\mathcal{T}_{2}$ and since $\mathcal{T}_{2} \subset V+W$ we get that $p$ is in $V+W$ as well. Hence all the points in $\mathcal{K}_{3}$ are in $V+W$. As $W_{2} \cup W_{3} \cup \mathcal{K}_{2} \cup \mathcal{K}_{3} \subset V+W$ it follows that also $\mathcal{K}_{1} \subset V+W$. The claim about the dimension of $V+W$ is clear.

This concludes the proof of the second case and with it the proof of Theorem 26.

\section{Structure theorem for quadratics satisfying $Q \in \sqrt{\left(Q_{1}, Q_{2}\right)}$}

An important tool in the proofs of our main results is the following theorem that classifies all the possible cases in which a quadratic $Q$ is in the radical of two other quadratics, where all quadratics are irreducible.

Before stating the theorem we explain the intuition behind the different cases. We would like to understand when does a quadratic polynomial $Q$ can belong to the radical of two other quadratics. Clearly, if $Q$ is a linear combination of $Q_{1}, Q_{2}$ then it is in their radical (and in fact, in their linear span). Another option is that $Q_{2}=\alpha Q_{1}+b^{2}$ and then $Q$ can be of the form $\beta Q_{1}+b \cdot a$. This case is clearly different than the linear span case. Finally, another option is the following situation: $Q_{1}^{\prime}=x y, Q_{2}^{\prime}=z(x+z)$ and $Q^{\prime}=y z$. It is not hard to verify that in this case too, $Q^{\prime} \in \sqrt{\left(Q_{1}^{\prime}, Q_{2}^{\prime}\right)}$. All these polynomials are reducible of course, but by defining, e.g., $Q_{1}=Q_{1}^{\prime}+Q_{2}^{\prime}, Q_{2}=Q_{1}^{\prime}-Q_{2}^{\prime}$ and $Q=Q^{\prime}+Q_{1}^{\prime}+Q_{2}^{\prime}$ we get three irreducible polynomials that do not fall into any of the previous two cases. Thus, all the three cases are distinct and can happen. What Theorem 10 shows is that, essentially, these are the only possible cases. To ease the reading we repeat the theorem here with slightly different notation.

Theorem 29. Let $Q, Q_{1}, Q_{2}$ be such that $Q \in \sqrt{Q_{1}, Q_{2}}$. Then one of the following cases hold:

1. $Q$ is in the linear span of $Q_{1}, Q_{2}$

2. There exists a non trivial linear combination of the form $\alpha Q_{1}+\beta Q_{2}=b^{2}$ where $b$ is a linear function

3. There exist two linear functions $b_{1}$ and $b_{2}$ such that when setting $b_{1}=b_{2}=0$ we get that $Q, Q_{1}$ and $Q_{2}$ vanish. In other words, $Q, Q_{1}, Q_{2} \in \sqrt{\left(b_{1}, b_{2}\right)}$.

Proof. By applying a suitable linear transformation we can assume that for some $r \geq 1$

$$
Q_{1}=\sum_{i=1}^{r} x_{i}^{2}
$$


We can also assume wlog that $x_{1}^{2}$ appears only in $Q_{1}$ as we can replace $Q_{2}$ with any polynomial of the form $Q_{2}^{\prime}=Q_{2}-\alpha Q_{1}$ without affecting the result. Indeed, $Q \in \sqrt{\left(Q_{1}, Q_{2}\right)}$ if and only if $Q \in \sqrt{\left(Q_{1}, Q_{2}^{\prime}\right)}$. Furthermore, all cases in the theorem remain the same if we replace $Q_{2}$ with $Q_{2}^{\prime}$ and vice versa.

In a similar fashion we can replace $Q$ with $Q^{\prime}=Q-\beta Q_{1}$ to get rid of the term $x_{1}^{2}$ in $Q$. Thus, wlog, the situation is

$$
\begin{aligned}
Q_{1} & =x_{1}^{2}+Q_{1}^{\prime} \\
Q_{2} & =x_{1} \cdot b_{2}-A \\
Q & =x_{1} \cdot b+B
\end{aligned}
$$

where $Q_{1}^{\prime}, A, B, b_{2}$ and $b$ do not depend on $x_{1}$.

The first case we handle is when the "new" $Q_{2}$ does not depend on $x_{1}$.

Claim 31. If $b_{2}=0$ then Case 2 of the theorem holds.

Proof. For any assignment satisfying $A=0$ there are two solutions to $Q_{1}=0$, unless $Q_{1}^{\prime}=0$, whereas $Q$ vanishes for only one value of $x_{1}$. Thus, we must have $Q_{1}^{\prime}=0$ modulo $A$, which means that either $A$ is a square of a linear function and so $Q_{1}$ and $Q_{2}$ satisfy Case 2 of the theorem (as we assume $b_{2}=0$ ), or $Q_{1}=\alpha \cdot A$ for some nonzero constant $\alpha$ and then $x_{1}^{2}$ is in the span of $Q_{1}$ and $Q_{2}$, and again Case 2 of the theorem holds.

We next handle the case where the "new" $Q_{2}$ is reducible.

Claim 32. If $b_{2}$ divides $A$ then the conclusion of the theorem holds.

Proof. If $b_{2}$ divides $A$ then $Q_{2}=b_{2} \cdot b_{2}^{\prime}$. Assume that $b_{2}^{\prime}$ is not a constant multiple of $b_{2}$ (as otherwise Case 2 of the theorem holds). Then, after a suitable invertible linear transformation we have $Q_{2}=y \cdot z$. Denote

$$
Q_{1}=\alpha y^{2}+\beta z^{2}+y \cdot \ell_{1}+z \cdot \ell_{2}+Q_{1}^{\prime \prime}
$$

and

$$
Q=\alpha^{\prime} y^{2}+\beta^{\prime} z^{2}+\gamma^{\prime} y z+y \cdot k_{1}+z \cdot k_{2}+Q^{\prime \prime},
$$

where $\ell_{1}, \ell_{2}, k_{1}, k_{2}, Q_{1}^{\prime \prime}, Q^{\prime \prime}$ do not involve $y$ nor $z$. Observe that since we can subtract a multiple of $Q_{2}$ from $Q_{1}$ we can assume that the term $y z$ does not appear in $Q_{1}$. Consider the assignment $y=0$. This simplifies $Q_{1}$ and $Q$ to:

$$
\left.Q_{1}\right|_{y=0}=\beta z^{2}+z \cdot \ell_{2}+Q_{1}^{\prime \prime}
$$

and

$$
\left.Q\right|_{y=0}=\beta^{\prime} z^{2}+z \cdot k_{2}+Q^{\prime \prime},
$$

which are two polynomials not depending on $y$. We now have that any assignment that makes $\left.Q_{1}\right|_{y=0}$ vanish, also makes $\left.Q\right|_{y=0}$ vanish. In other words $\left.Q\right|_{y=0} \in \sqrt{\left(\left.Q_{1}\right|_{y=0}\right)}$. This means that all irreducible factors of $\left.Q_{1}\right|_{y=0}$ divide $\left.Q\right|_{y=0}$. Thus, either $\left.Q\right|_{y=0}=\left.\delta \cdot Q_{1}\right|_{y=0}$ for some constant $\delta$, or $\left.Q_{1}\right|_{y=0}=b_{3}^{2}$ and $\left.Q\right|_{y=0}=b_{3} \cdot b_{3}^{\prime}$ for some linear functions $b_{3}, b_{3}^{\prime}$.

Notice that in the second case, if we set $y=b_{3}=0$ then $Q_{1}$ and $Q_{2}$ vanish and hence $Q$ also vanishes and Case 3 of the theorem holds. 


\section{SYLVESTER-GALLAI TYPE THEOREMS FOR QUADRATIC POLYNOMIALS}

So let us assume that $\left.Q_{1}\right|_{y=0}$ divides $\left.Q\right|_{y=0}$. We repeat the same reasoning when setting $z=0$ and again assume that $\left.Q_{1}\right|_{z=0}$ divides $\left.Q\right|_{z=0}$. By comparing coefficients we get that there are constants $\delta, \delta^{\prime}$ such that $\beta^{\prime}=\delta \beta, k_{2}=\delta \ell_{2}, Q^{\prime \prime}=\delta Q_{1}^{\prime \prime}$ and $\alpha^{\prime}=\delta^{\prime} \alpha, k_{1}=\delta^{\prime} \ell_{1}, Q^{\prime \prime}=\delta^{\prime} Q_{1}^{\prime \prime}$. It follows that either $\delta=\delta^{\prime}$ and we obtain that $Q=\delta Q_{1}+\gamma^{\prime} Q_{2}$, which satisfies Case 1 of the theorem or that $Q_{1}^{\prime \prime}=Q_{1}=0$ in which case $Q_{1}, Q_{2}, Q$ all vanish when setting $y=z=0$ as in Case 3 of the theorem.

Hence, from now on we assume that $b_{2}$ is non-zero and does not divide $A$. Consider the resultant of $Q_{1}, Q_{2}$ (as given in Equation 30) with respect to $x_{1}$. It is equal to

$$
\operatorname{Res}_{x_{1}}\left(Q_{1}, Q_{2}\right)=A^{2}+b_{2}^{2} \cdot Q_{1}^{\prime} .
$$

We next study what happens when the resultant vanishes. I.e. when

$$
\operatorname{Res}_{x_{1}}\left(Q_{1}, Q_{2}\right)=A^{2}+b_{2}^{2} \cdot Q_{1}^{\prime}=0 .
$$

Claim 35. Whenever $\operatorname{Res}_{x_{1}}\left(Q_{1}, Q_{2}\right)=0$ it holds that $A \cdot b+b_{2} \cdot B=0$.

Proof. If $\operatorname{Res}_{x_{1}}\left(Q_{1}, Q_{2}\right)=0$ then either $b_{2}=0$, which also implies $A=0$ and in this case the claim clearly holds, or $b_{2} \neq 0$. Consider the case $b_{2} \neq 0$ and set $x_{1}=A / b_{2}$ (we are free to select a value for $x_{1}$ as $\operatorname{Res}_{x_{1}}\left(Q_{1}, Q_{2}\right)$ does not involve $\left.x_{1}\right)$. Notice that for this substitution we have that $Q_{2}=0$ and that

$$
\left.Q_{1}\right|_{x_{1}=A / b_{2}}=\left(A / b_{2}\right)^{2}+Q_{1}^{\prime}=\operatorname{Res}_{x_{1}}\left(Q_{1}, Q_{2}\right) / b_{2}^{2}=0 .
$$

Hence, we also have $\left.Q\right|_{x_{1}=A / b_{2}}=0$. In other words that

$$
A \cdot b+b_{2} \cdot B=0
$$

In other words, Claim 35 implies that

$$
A \cdot b+b_{2} \cdot B \in \sqrt{\left(\operatorname{Res}_{x_{1}}\left(Q_{1}, Q_{2}\right)\right)} .
$$

Thus, there exists an integer $k$ and a polynomial $\psi$ so that

$$
\left(A \cdot b+b_{2} \cdot B\right)^{k}=\psi \cdot \operatorname{Res}_{x_{1}}\left(Q_{1}, Q_{2}\right)=\psi \cdot\left(A^{2}+b_{2}^{2} \cdot Q_{1}^{\prime}\right) .
$$

This means that all irreducible factors of $A^{2}+b_{2}^{2} \cdot Q_{1}^{\prime}$ divide $A \cdot b+b_{2} \cdot B$. As $\operatorname{deg}\left(A^{2}+b_{2}^{2} \cdot Q_{1}^{\prime}\right)=4$ and $\operatorname{deg}\left(A \cdot b+b_{2} \cdot B\right)=3$ it follows, by examining the possible ways that a degree 4 polynomial can factor, that one of the following cases must hold:

1. There is a quadratic polynomial $C$ and a linear function $a$ such that

$$
\begin{gathered}
A^{2}+b_{2}^{2} \cdot Q_{1}^{\prime}=C^{2} \\
b \cdot A+b_{2} \cdot B=a \cdot C
\end{gathered}
$$


2. For some scalar $\lambda$, a linear function $a$ and a quadratic $C$

$$
\begin{array}{r}
A^{2}+b_{2}^{2} \cdot Q_{1}^{\prime}=a^{2} \cdot C \\
b \cdot A+b_{2} \cdot B=\lambda \cdot a \cdot C
\end{array}
$$

We next handle each of these cases.

Case 1: we have that

$$
b_{2}^{2} \cdot Q_{1}^{\prime}=C^{2}-A^{2}=(C+A)(C-A) .
$$

If $Q_{1}^{\prime}$ is irreducible then $\alpha b_{2}^{2}=(C+A)$ and $Q_{1}^{\prime}=\alpha(C-A)$, or $\alpha b_{2}^{2}=(C-A)$ and $Q_{1}^{\prime}=\alpha(C+A)$ for some $\alpha \neq 0$. In the first case we get that $Q_{1}^{\prime}=-2 \alpha A+\alpha^{2} b_{2}^{2}$ and hence $Q_{1}+2 \alpha Q_{2}=\left(x+\alpha b_{2}\right)^{2}$. Similarly, in the second case we get $Q_{1}^{\prime}=2 \alpha A+\alpha^{2} b_{2}^{2}$ and thus $Q_{1}-2 \alpha Q_{2}=\left(x-\alpha b_{2}\right)^{2}$. In either cases, Case 2 of the theorem holds.

If $Q_{1}^{\prime}$ is reducible, i.e. $Q_{1}^{\prime}=e \cdot f$, then either the analysis above continues to hold or it must be the case that (w.l.o.g.) $C+A=b_{2} \cdot e$ and $C-A=b_{2} \cdot f$. It follows that in this case $b_{2}$ divides $A$ and we are done by Claim 32 .

Case 2: From Equation 36 we learn that $a^{2} \mid \operatorname{Res}_{x_{1}}\left(Q_{1}, Q_{2}\right)$ so in particular, when setting $a=0$ we get that the resultant is zero. Theorem 12 implies that, modulo $a$, either one of $Q_{1}, Q_{2}$ vanishes, or that $Q_{1}$ and $Q_{2}$ share a linear factor.

As $a$ does not involve $x_{1}$, clearly $\left.Q_{1}\right|_{a=0} \neq 0$. Further, for $Q_{2}$ to vanish modulo $a$ we need that $b_{2}$ is a multiple of $a$, and vice versa. This implies that $b_{2}$ divides $A$ and we are done by Claim 32 .

We thus have to deal with the case that, modulo $a, Q_{1}$ and $Q_{2}$ share a linear factor. Let $a^{\prime}$ be that common linear factor. We get that by setting $a=a^{\prime}=0$ both $Q_{1}$ and $Q_{2}$ vanish and hence also $Q$ vanishes and Case 3 of the theorem holds.

This concludes the proof of Theorem 29.

\section{Sylvester-Gallai theorem for quadratic polynomials}

In this section we prove Theorem 7. For convenience we repeat the statement of the theorem.

Theorem (Theorem 7). Let $\left\{Q_{i}\right\}_{i \in[m]}$ be homogeneous quadratic polynomials such that each $Q_{i}$ is either irreducible or a square of a linear function. Assume further that for every $i \neq j$ there exists $k \notin\{i, j\}$ such that $Q_{k} \in \sqrt{\left(Q_{i}, Q_{j}\right)}$. Then the linear span of the $Q_{i}$ 's has dimension $O(1)$.

Remark 37. The requirement that the polynomials are homogeneous is not essential as homogenization does not affect the property $Q_{k} \in \sqrt{\left(Q_{i}, Q_{j}\right)}$. 


\subsection{Some useful claims}

In this section we look at some implications of Theorem 29. We do so by considering two irreducible polynomials $Q_{1}$ and $Q_{2}$ and consider sets of polynomials that satisfy Case 2 or Case 3 of Theorem 29 with $Q_{1}$ and $Q_{2}$.

Claim 38. Let $Q_{1}, Q_{2}$ be two linearly independent quadratic polynomials and let $F_{1}, \ldots, F_{m}$ be quadratic polynomials such that for every $i$ there exist linear functions $\ell_{i}, b_{i}$ and a scalar $\beta_{i}$ so that

$$
F_{i}=Q_{1}+\ell_{i}^{2}=\beta_{i} \cdot Q_{2}+b_{i}^{2}
$$

Then, there exists a 4-dimensional space $V$ such that for every $i,\left\{\ell_{i}, b_{i}\right\} \subseteq V$.

Proof. If $m \leq 2$ then the claim is trivial. We consider two cases.

1. Case 1: For all $i, \beta_{i}=\beta_{1}$ :

Let $V=\operatorname{span}\left\{b_{1}, \ell_{1}\right\}$. From the two representations of $F_{1}$ we get that

$$
Q_{1}-\beta_{1} Q_{2}=b_{1}^{2}-\ell_{1}^{2}=\left(b_{1}-\ell_{1}\right) \cdot\left(b_{1}+\ell_{1}\right) \neq 0,
$$

where the fact that the expression above is nonzero follows as $Q_{1}$ and $Q_{2}$ are linearly indepdent. Similarly, by considering the two representations of $F_{j}$ we get that

$$
Q_{1}-\beta_{1} Q_{2}=b_{j}^{2}-\ell_{j}^{2}=\left(b_{j}-\ell_{j}\right) \cdot\left(b_{j}+\ell_{j}\right) .
$$

Thus,

$$
\left(b_{1}-\ell_{1}\right) \cdot\left(b_{1}+\ell_{1}\right)=\left(b_{j}-\ell_{j}\right) \cdot\left(b_{j}+\ell_{j}\right) .
$$

Unique factorization implies that $b_{j}, \ell_{j} \in \operatorname{span}\left\{b_{i}, \ell_{1}\right\}=V$ as claimed.

2. Case 2: There is $j$ such that $\beta_{j} \neq \beta_{1}$ :

In this case we have that

$$
\begin{aligned}
& F_{1}=Q_{1}+\ell_{1}^{2}=\beta_{1} Q_{2}+b_{1}^{2}, \\
& F_{j}=Q_{1}+\ell_{j}^{2}=\beta_{j} Q_{2}+b_{j}^{2},
\end{aligned}
$$

and the matrix

$$
\left[\begin{array}{ll}
1 & -\beta_{1} \\
1 & -\beta_{j}
\end{array}\right]
$$

is invertible. It follows that

$$
Q_{2}, Q_{1} \in \operatorname{span}\left\{\left(b_{1}-\ell_{1}\right) \cdot\left(b_{1}+\ell_{1}\right),\left(b_{j}-\ell_{j}\right) \cdot\left(b_{j}+\ell_{j}\right)\right\} .
$$

Let

$$
V=\operatorname{span}\left\{b_{1}, b_{j}, \ell_{1}, \ell_{j}\right\}
$$

Consider any index $k$. W.l.o.g. $\beta_{k} \neq \beta_{j}$. Thus, as before, we get that

$$
Q_{1} \in \operatorname{span}\left\{\left(b_{j}-\ell_{j}\right) \cdot\left(b_{j}+\ell_{j}\right),\left(b_{k}-\ell_{k}\right) \cdot\left(b_{k}+\ell_{k}\right)\right\} \text {. }
$$




\section{AMIR SHPILKA}

Hence, either $Q_{1}=\alpha \cdot\left(b_{j}-\ell_{j}\right) \cdot\left(b_{j}+\ell_{j}\right)$ or, for some $\alpha_{3}$ and a nonzero $\alpha_{4}$,

$$
Q_{1}=\alpha_{3} \cdot\left(b_{j}-\ell_{j}\right) \cdot\left(b_{j}+\ell_{j}\right)+\alpha_{4} \cdot\left(b_{k}-\ell_{k}\right) \cdot\left(b_{k}+\ell_{k}\right) .
$$

We first handle the later case. Combining Equation 42 with Equation 41 we get that there exist constants $\alpha_{1}, \ldots, \alpha_{4}$, with $\alpha_{4} \neq 0$ so that

$\alpha_{1} \cdot\left(b_{1}-\ell_{1}\right) \cdot\left(b_{1}+\ell_{1}\right)+\alpha_{2} \cdot\left(b_{j}-\ell_{j}\right) \cdot\left(b_{j}+\ell_{j}\right)=\alpha_{3} \cdot\left(b_{j}-\ell_{j}\right) \cdot\left(b_{j}+\ell_{j}\right)+\alpha_{4} \cdot\left(b_{k}-\ell_{k}\right) \cdot\left(b_{k}+\ell_{k}\right)$.

By switching sides it is easy to see that both $b_{k}-\ell_{k}$ and $b_{k}+\ell_{k}$ are spanned by the functions in $V$. In the former case where $Q_{1}=\alpha \cdot\left(b_{j}-\ell_{j}\right) \cdot\left(b_{j}+\ell_{j}\right)$, it follows from Equation 39 (for $i=j$ ) that $Q_{2}=\frac{\alpha_{1}-1}{\beta_{j}} \cdot\left(b_{j}-\ell_{j}\right) \cdot\left(b_{j}+\ell_{j}\right)$. This contradicts the assumption that $Q_{1}, Q_{2}$ are linearly independent.

Corollary 43. Under the hypothesis of Claim 38, there exist four linear functions $a_{1}, a_{2}, a_{3}, a_{4}$ such that every $F_{i}$ is a linear combination of $Q_{1},\left\{a_{i} \cdot a_{j}\right\}_{i \leq j}$.

Proof. Let $V$ be the subspace guaranteed by Claim 38. Let $\left\{a_{1}, \ldots, a_{4}\right\}$ be such that $V=$ $\operatorname{span}\left\{a_{1}, a_{2}, a_{3}, a_{4}\right\}$. The claim follows immediate from the fact that each $\ell_{i}$ is a linear combination of $a_{1}, a_{2}, a_{3}, a_{4}$.

Claim 44. Let $F_{1}, \ldots, F_{m^{\prime}}$ be quadratics in our set ${ }^{8}$ that satisfy Case 3 of Theorem 29 with an irreducible $Q$. Then there exists an $O(1)$-dimensional space $V$ such that each $F_{i}$ is a quadratic polynomial in the linear functions in $V$.

Proof. As $Q$ satisfies Case 3 of Theorem 29 and is irreducible it follows that $\operatorname{rank}_{s}(Q)=2$ (recall Definition 13). Thus, $Q$ is a quadratic polynomial in at most 4 linear functions. Let $V$ to be the space spanned by the linear functions in a minimal representation of $Q$. By Claim 14 it follows that $V$ is well defined. Clearly $\operatorname{dim}(V) \leq 4$.

Let $z$ be a new variable. Set each basis element of $V$ to a random multiple of $z$ (say by picking the multiples independently uniformly at random from $[0,1]$ ). Each $F_{i}$ now becomes $z \cdot b_{i}$ for some nonzero $b_{i}$. Indeed, if we further set $z=0$ then all linear functions in the representation of $Q$ vanish and hence also $F_{i}$ vanishes (this again follows from Claim 14). Further, $b_{i} \neq 0$ as we mapped the basis elements to random multiples of $z$. We next show that unless all linear functions in the minimal representation of $F_{i}, F_{j}$ are in $V$ then $F_{i}, F_{j}$ remain linearly independent after this restriction.

Claim 45. Let $V$ be a linear space of linear functions. Let $F=v_{1} \cdot \ell_{1}+v_{2} \cdot \ell_{2}$ and $G=v_{3} \cdot \ell_{3}+v_{4} \cdot \ell_{4}$ be two linearly independent irreducible quadratics, where for every $i, v_{i} \in V$. If $\operatorname{span}\left\{\ell_{1}, \ldots, \ell_{4}\right\} \nsubseteq V$ then with probability $1, F$ and $G$ remain linearly independent even after we map the basis elements of $V$ to random multiples of a new variable $z$ (say, by picking the multiples uniformly and independently from the segment $[0,1])$.

\footnotetext{
${ }^{8}$ I.e. they are a subset of the $\left\{Q_{i}\right\}$ from the statement of Theorem 7.
} 
We postpone the proof of Claim 45 and continue with the proof of Claim 44 . We next show that the linear functions $\left\{b_{i}\right\}_{i} \cup\{z\}$ satisfy the "usual" Sylvester-Gallai condition, i.e., that any two of them span a third function in the set (with the possible exception of $z$ ). In fact, we will add to this set all quadratics in our set that are now of the form $z \cdot \ell$ for a linear $\ell$.

Consider two quadratics $Q_{1}=z b_{1}, Q_{2}=z b_{2}$ so that neither $b_{1}$ nor $b_{2}$ is a multiple of $z$. If $\left\{b_{1}, b_{2}\right\}$ span $z$ then we are done. Otherwise, assume that $Q_{3}$ vanishes when $Q_{1}$ and $Q_{2}$ vanish. Then clearly $z$ divides $Q_{3}$. Thus $Q_{3}=z b_{3}$ and $b_{3}$ is in our set. Further, when we set $b_{1}=b_{2}=0$ both $Q_{1}$ and $Q_{2}$ vanish and hence $Q_{3}$ vanishes as well. Since $z \notin \operatorname{span}\left\{b_{1}, b_{2}\right\}$ this implies that $b_{3} \in \operatorname{span}\left\{b_{1}, b_{2}\right\}$ and in this case too $b_{1}$ and $b_{2}$ span a third linear function in $\left\{b_{i}\right\}_{i} \cup\{z\}$. Note also that, by Claim $45, b_{3}$ is not a multiple of $b_{1}$ nor of $b_{2}$ as this would imply that $Q_{3}$ and $Q_{1}$ (or $Q_{2}$ ) are linearly dependent in contradiction to our assumption.

From Corollary 16 (recalling Remark 17) we get that the dimension of all those $\left\{b_{i}\right\}_{i}$ is $O(1)$.

We now repeat the same argument again for a different random mapping of the basis elements of $V$ to multiples of $z$. As before each $F_{i}$ is mapped to a polynomial of the form $z \cdot b_{i}^{\prime}$ and again the dimension of $\left\{b_{i}^{\prime}\right\}_{i}$ is $O(1)$. Let $U$ be the subspace containing the span of $V \cup\left\{b_{i}\right\}_{i} \cup\left\{b_{i}^{\prime}\right\}_{i}$. Clearly $\operatorname{dim}(U)=O(1)$. We next show that every $F_{i}$ is a polynomial in the linear functions in $U$. Indeed, let $F=v_{1} \cdot u_{1}+v_{2} \cdot u_{2}$ be arbitrary polynomial from $\left\{F_{i}\right\}_{i}$, where $v_{1}, v_{2} \in V$. Assume the first mapping mapped $v_{i} \mapsto \alpha_{i} \cdot z$ and the second mapping is $v_{i} \mapsto \beta_{i} \cdot z$. Then, $F$ was mapped to $z \cdot b$ under the first mapping where $b=\alpha_{1} u_{1}+\alpha_{2} u_{2}$ and to $z \cdot b^{\prime}$ under the second mapping where $b^{\prime}=\beta_{1} u_{1}+\beta_{2} u_{2}$. As $\alpha_{1}, \alpha_{2}, \beta_{1}, \beta_{2}$ where chosen uniformly independently at random from $[0,1]$ it follows that the determinant

$$
\left|\left[\begin{array}{ll}
\alpha_{1} & \alpha_{2} \\
\beta_{1} & \beta_{2}
\end{array}\right]\right| \neq 0
$$

and hence $u_{1}, u_{2} \in \operatorname{span}\left\{b, b^{\prime}\right\} \subseteq U$. As we also have $v_{1}, v_{2} \in V \subseteq U$ the claim follows.

This concludes the proof of Claim 44.

We now give the proof of Claim 45.

Proof of Claim 45 . Let $x_{1}, \ldots, x_{k}$, for some $1 \leq k \leq 4$ be a basis for $\operatorname{span}\left\{\ell_{1}, \ldots, \ell_{4}\right\}$ such that for some $0<t \leq k, x_{t+1}, \ldots, x_{k}$ for a basis to $\operatorname{span}\left\{\ell_{1}, \ldots, \ell_{4}\right\} \cap V$. We can rewrite $F$ and $G$ as

$$
F=\sum_{i=1}^{t} x_{i} u_{i}+F^{\prime} \quad \text { and } \quad G=\sum_{i=1}^{t} x_{i} w_{i}+G^{\prime}
$$

where $u_{i}, w_{i} \in V$ and $F^{\prime}, G^{\prime}$ are defined over $V$, and, w.l.o.g., for every $i$, at least one of $u_{i}$ and $w_{i}$ is nonzero. Observe that $F$ and $G$ are linearly independent (over $\mathbb{C}$ ) if and only if the two vectors

$$
u_{F}=\left(u_{1}, \ldots, u_{t}, F^{\prime}\right) \quad \text { and } \quad w_{G}=\left(w_{1}, \ldots, w_{t}, G^{\prime}\right)
$$

are linearly independent over $\mathbb{C}(V)$, the function field generated by adding the linear functions in $V$ to $\mathbb{C}$. Indeed, if $F$ and $G$ are linearly dependent over $\mathbb{C}$ then clearly $u_{F}$ and $w_{G}$ are linearly dependent over $\mathbb{C}$, and hence over $\mathbb{C}(V)$. If on the other hand $u_{F}$ and $w_{G}$ are linearly dependent over $\mathbb{C}(V)$ then this means that for some polynomials $f(V)$ and $g(V)$ we have

$$
f \cdot\left(u_{1}, \ldots, u_{t}, F^{\prime}\right)=g \cdot\left(w_{1}, \ldots, w_{t}, G^{\prime}\right) .
$$


This implies that all the $2 \times 2$ determinants vanish, i.e. that $u_{i} \cdot w_{j}-u_{j} \cdot w_{i}=0$, for every $i$ and $j$, and $u_{i} \cdot G^{\prime}-w_{i} \cdot F^{\prime}=0$. By unique factorization we get that there are two possible cases:

1. There is $\alpha \in \mathbb{C}$ so that $u_{i}=\alpha w_{i}$ for some $i$ : The equality $u_{i} \cdot w_{j}-u_{j} \cdot w_{i}=0$ implies that for every $j$ we actually have $u_{j}=\alpha w_{j}$, and the fact that $u_{i} \cdot G^{\prime}-w_{i} \cdot F^{\prime}=0$ gives $F^{\prime}=\alpha G^{\prime}$ and thus $u_{F}$ and $w_{G}$ are linearly dependent over $\mathbb{C}$ and indeed $F$ and $G$ are linearly dependent.

2. There are constants $\alpha_{i}$ such that for every $i, u_{i}=\alpha_{i} u_{1}$ and $w_{i}=\alpha_{i} w_{1}$ : In this case, since $F$ is irreducible, it holds that $u_{1}$ does not divide $F^{\prime}$. As $u_{1} \cdot G^{\prime}-w_{1} \cdot F^{\prime}=0$, by unique factorization it follows that $u_{1}$ is a multiple of $w_{1}$ and we are thus in the previous case again.

It therefore follows that the matrix

$$
M=\left[\begin{array}{llll}
u_{1} & \ldots & u_{j} & F^{\prime} \\
w_{1} & \ldots & w_{j} & G^{\prime}
\end{array}\right]
$$

is full rank over $\mathbb{C}(V)$. Thus the determinant of ${ }^{9} M \cdot M^{\dagger}$ is a nonzero polynomial over $V$. The SchwartzZippel-DeMillo-Lipton lemma now implies that sending each basis element of $V$ to a random multiple of $z$ will make the determinant nonzero with probability 1 . This also means that $F$ and $G$ remain linear independent after such mapping.

\subsection{An important special case}

Before proving Theorem 7, we prove a special case where there is a set of quadratics J, and a vector space of linear forms $V$, and each quadratic in our set is a linear combination of quadratics from $\mathcal{J}$ and a quadratics defined over $V$, and, all nonzero polynomials in the span of $\mathcal{J}$ remain of rank at least 2 even when we set the functions in $V$ to zero. We show that in this case the linear forms in $\mathcal{L}$ satisfy the Sylvester-Gallai condition among themselves.

Claim 46. Let $Q \cup \mathcal{L}$ satisfy the assumption of Theorem 7 where

1. 2 consists of irreducible quadratics.

2. There is a set of polynomials $\mathcal{J}$ and an $O(1)$-dimensional space $V$ such that every polynomial in 2 is in the linear span of $\mathcal{J}$ and quadratics over $V$. Furthermore, no nonzero linear combination of the polynomials in J can be expressed as $x a+y b+F(V)$ where $F$ is any quadratic over $V$ and $x, a, b, y$ are any four linear forms.

\section{3. $\mathcal{L}$ is a set of squares of linear functions.}

Then, the dimension of the space spanned by the functions whose squares are in $\mathcal{L}$ has dimension $O(1)$.

Proof. Denote $\mathcal{L}^{\prime}=\mathcal{L} \backslash V$. We shall prove that the linear functions in $\mathcal{L}^{\prime}$ satisfy the Sylvester-Gallai condition and hence their span has dimension $O(1)$ as claimed.

Let $x, y \in \mathcal{L}^{\prime}$. Let $Q$ be such that $Q \in \sqrt{(x, y)}$. Thus, there exist linear functions $a, b$ so that $Q=x a+y b$. We next consider two cases for $Q$.

\footnotetext{
${ }^{9} M^{\dagger}$ is the conjugate transpose of $M$.
} 
If $Q \in \mathcal{Q}$ then $Q=Q^{\prime}+G(V)$, where $Q^{\prime}$ is a linear combination of the polynomials in J. In particular, $Q^{\prime}=x a+y b-G(V)$. This implies that $Q^{\prime}=0$ as otherwise we get a contradiction to the assumptions on $\mathcal{J}$ and $V$. Hence, $x a+y b=Q=G(V)$. As $Q$ is irreducible it must hold that $x, y \in V$ (by Claim 14). This is in contradiction to the definition of $\mathcal{L}^{\prime}$.

The remaining case is when $Q \in \mathcal{L}$. Thus, $Q=\ell^{2}$ for some linear $\ell$, and it follows that $\ell \in \operatorname{span}\{x, y\}$. Note however that we may have $\ell \in V$. To overcome this we apply a random projection to the linear functions in $V$ so that they are all equal to some multiple of a new variable $z$. As before it is not hard to see that even after this projection any two linear functions from $\mathcal{L}^{\prime}$ are projected to linearly independent linear functions. Hence, in the case above, there is a third linear function in $\mathcal{L}^{\prime} \cup\{z\}$ that is spanned by $x, y$. It follows that $\mathcal{L}^{\prime} \cup\{z\}$ satisfy the conditions of Corollary 16 (with, say, $\delta=1 / 2$ ) and hence $\operatorname{dim}\left(\mathcal{L}^{\prime}\right)=O(1)$ as claimed.

\subsection{The proof}

We are now ready to prove Theorem 7. The proof follows the outline sketched in subsection 1.2 and it relies on the claims proved in subsection 5.1 and on Corollary 16.

Proof of Theorem 7. Partition the polynomials to two sets. Let $\mathcal{L}$ be the set of all squares and let $\mathcal{Q}$ be the subset of irreducible quadratics. Denote $|\mathcal{Q}|=m_{1}$.

We next focus on polynomials in $Q$. We prove that they are contained in an $O(1)$-dimensional space of a special form.

Call a polynomial $Q \in \mathcal{Q}$ bad if there are less than, say, $m_{1} / 100$ pairs $\left(Q_{1}, Q_{2}\right) \in \mathcal{Q} \times \mathcal{Q}$ so that

$Q_{2} \in \sqrt{\left(Q, Q_{1}\right)}$ and $Q, Q_{1}$ satisfy item 1 of Theorem 29 (i.e. $Q_{2}$ is in their linear span). If $Q \in \mathcal{Q}$ is not bad then we call it a good polynomial. We handle two cases according to whether there is at most one bad polynomial or more than that.

\section{There is at most one bad polynomial:}

In this case, from Corollary 16 we get that the linear span of the polynomials in $Q$ has dimension $O(1)$.

Assume $Q_{1}, \ldots, Q_{k}$ for some $k=O(1)$ span $Q$. We now repeat the following process. We start with $\mathcal{J}=\left\{Q_{1}, \ldots, Q_{k}\right\}$ and $V=\emptyset$. If there is some nontrivial linear combination of the polynomials in $\mathcal{J}$ that is equal to a quadratic of the form $a_{1} b_{1}+a_{2} b_{2}$, where $a_{i}, b_{i}$ are linear functions then we add $a_{1}, a_{2}, b_{1}, b_{2}$ to $V$ and remove one of the polynomials that participated in the linear combination from $\mathcal{J}$. We continue doing so according to the following rule. If there exists a linear combination of the polynomials in $\mathcal{J}$ that is equal to a polynomial of the form $F(V)+a b+a^{\prime} b^{\prime}$, where $F(V)$ is a quadratic polynomial over linear functions in $V$, then we add $a, b, a^{\prime}, b^{\prime}$ to $V$ and remove some polynomial participating in the linear combination from $\mathcal{J}$. We do so until no such linear combination exists or until J is empty. At the end $|V| \leq 4 k=O(1)$. Abusing notation we now think of $V$ as the space spanned by the linear functions in it. Clearly $\operatorname{dim}(V) \leq 4 k=O(1)$.

The argument above implies that the conditions of Claim 46 are satisfied by our $\mathcal{J}, V, \mathcal{Q}$ and $\mathcal{L}$. We thus obtain that $\operatorname{dim}(\mathcal{L})=O(1)$. Combined with the fact that $|\mathcal{J}|=O(1)$ this completes the proof for the case when there is at most one bad polynomial. We handle the other case next. 


\section{There are at least two bad polynomials:}

Claim 47 (At least two bad polynomials). If 2 contains at least two bad polynomials, $Q_{1}$ and $Q_{2}$, then there is a space $V$ of linear functions of dimension $O(1)$ so that every polynomial in $Q$ is a linear combination of $Q_{1}$ and a quadratic over $V$.

Proof. Notice that for $Q_{1}$ there are $0.99 m_{1}$ polynomials in $Q$ that even together with $Q_{1}$ do not span any other polynomial in $\mathcal{Q}$. The same holds for $Q_{2}$. Consider a polynomial $Q_{j}$ so that $Q_{1}$ and $Q_{j}$ do not span any other polynomial in 2 . We conclude that $Q_{1}$ and $Q_{j}$ satisfy Case 2 or Case 3 of Theorem 29. Indeed, if $Q_{1}$ and $Q_{j}$ satisfy Case 1 of Theorem 29 then they span some polynomial in $\mathcal{L}$ and in particular they span a square, but this means that they also satisfy Case 2 of Theorem 29.

From the discussion above it follows that there are at least $0.98 m_{1}$ polynomials in $Q$ satisfying Case 2 or Case 3 of the theorem with $Q_{1}$ and $Q_{2}$. Let $\mathcal{F}$ be the set of these polynomials. Partition $\mathcal{F}$ to three sets $\mathcal{J}, \mathcal{J}, \mathcal{K}$ so that those polynomials in $\mathcal{J}$ satisfy Case 3 of Theorem 29 with $Q_{1}$, those in $\mathcal{J}$ satisfy Case 3 of Theorem 29 with $Q_{2}$ and those in $\mathcal{K}$ satisfy Case 2 of Theorem 29 with both $Q_{1}$ and $Q_{2}$. From Corollary 43 and Claim 44 we conclude that there is a an $O(1)$-dimensional space $V^{\prime}$ of linear functions such that all those $0.98 m_{1}$ polynomials are in the linear span of quadratics over $V^{\prime}$ and $Q_{1}$.

To simplify things further, if it is the case that $Q_{1}=F\left(V^{\prime}\right)+a a^{\prime}+b b^{\prime}$, i.e. that $Q_{1}$ can be written as a quadratic over $V^{\prime}$ plus two products of linear forms, then we add $a, a^{\prime}, b, b^{\prime}$ to $V^{\prime}$ and we do not consider $Q_{1}$ any more. ${ }^{10}$

We now consider the remaining $0.02 m_{1}$ polynomials in $Q$. In fact, consider those polynomials that cannot be spanned by quadratics over $V^{\prime}$ and $Q_{1}$ and call this set $\mathcal{F}^{c}$ (abusing notation).

Claim 48. For each $Q \in \mathcal{F}^{c}$ there are at least $0.96 m_{1}$ polynomials in $\mathcal{F}$ that satisfy either Case 2 or Case 3 of Theorem 29 with $Q$.

Proof. If $Q$ and $F \in \mathcal{F}$ span a polynomial in $\mathcal{L}$ then we say that $Q$ satisfies Case 2 with $F$. Thus, if $Q$ and $F \in \mathcal{F}$ satisfy item 1 of Theorem 29 then the third polynomial is not in $\mathcal{F}$ (as by switching sides we will get that $Q$ is also in $\mathcal{F}$ ). Hence, this polynomial must be in $\mathcal{F}^{c}$. Assume that $Q^{\prime}$ is this polynomial. Notice that there is no other $F^{\prime} \in \mathcal{F}$ that together with $Q$ spans $Q^{\prime}$ as in such a case $Q$ would be in $\mathcal{F}$. Indeed, let $\alpha_{1} Q+F=Q^{\prime}$ and $\alpha_{2} Q+F^{\prime}=Q^{\prime}$. Since $F$ and $F^{\prime}$ are linearly independent we get that $0 \neq\left(\alpha_{1}-\alpha_{2}\right) Q=F^{\prime}-F$ in contradiction to the assumption that $Q$ is in $\mathcal{F}^{c}$. Thus, $Q$ can satisfy item 1 of Theorem 29 with at most $\left|\mathcal{F}^{c}\right| \leq 0.02 m_{1}$ polynomials. It follows that there are at least $0.96 m_{1}$ polynomials in $\mathcal{F}$ that satisfy either Case 2 or Case 3 of Theorem 29 with $Q$.

We next show that all polynomials $Q \in \mathcal{F}^{c}$ satisfy Case 3 of Theorem 29 with some polynomial in $\mathcal{F}$. Indeed, if this is not the case then there must be a polynomial $Q$ that satisfy Case 2 of Theorem 29 with all polynomial in $\mathcal{F}$. Let $F_{1}, F_{2} \in \mathcal{F}$. Then, after rescaling, there are $a_{1}, a_{2}$ so that

\footnotetext{
${ }^{10}$ This step is not crucial at this point, it just makes some later argument a bit simpler.
} 
$Q+a_{1}^{2}=F_{1}$ and $Q+a_{2}^{2}=F_{2}$. Hence, $a_{1}^{2}-a_{2}^{2}=F_{2}-F_{1}$. As $F_{2}-F_{1}$ is a linear combination of $Q_{1}$ and quadratics over $V^{\prime}$, it must be the case that $F_{2}-F_{1}$ are defined over $V^{\prime}$ alone as otherwise we would have replaces $Q_{1}$ with two linear functions as described above. Thus, $a_{1}^{2}-a_{2}^{2}=F\left(V^{\prime}\right)$ and it follows that $a_{1}, a_{2} \in V^{\prime}$ and hence $Q \in \mathcal{F}$ in contradiction.

We now bound the dimension of $\mathcal{F}^{c}$. By an argument similar to the proof of Claim 44 it follows that there is an $O(1)$-dimensional space of linear functions, $V^{\prime \prime}$ such that all polynomials in $\mathcal{F}^{c}$ are quadratics over $V^{\prime \prime}$ : We send $V^{\prime}$ to a random multiple of a new variable $z$. This makes all polynomials in $\mathcal{F}^{c}$ to be of the form $z b_{i}$ and as before the linear functions $\left\{b_{i}\right\}_{i} \cup\{z\}$ satisfy the usual Sylvester-Gallai condition and we conclude using Corollary 16 (as in the proof of Claim 44 we repeat this twice for two independent mappings etc.). Set $V$ be the span of $V^{\prime \prime} \cup V^{\prime}$. This completes the proof of Claim 47

It remains to bound the dimension of $\mathcal{L}$. This however, follows immediately from Claim 46.

This concludes the proof of the case of two bad polynomials and with it the proof of Theorem 7.

\section{Edelstein-Kelly theorem for quadratic polynomials}

In this section we prove Theorem 8 . We repeat its statement for convenience.

Theorem (Theorem 8). Let $\mathcal{T}_{1}, \mathcal{T}_{2}$ and $\mathcal{T}_{3}$ be finite sets of homogeneous quadratic polynomials over $\mathbb{C}$ satisfying the following properties:

- Each $Q \in \cup_{i} \mathcal{T}_{i}$ is either irreducible or a square of a linear function.

- No two polynomials are multiples of each other (i.e., every pair is linearly independent).

- For every two polynomials $Q_{1}$ and $Q_{2}$ from distinct sets there is a polynomial $Q_{3}$ in the third set such that $Q_{3} \in \sqrt{\left(Q_{1}, Q_{2}\right)}$.

Then the linear span of the polynomials in $\cup_{i} \mathcal{T}_{i}$ 's has dimension $O(1)$.

Remark 49. As before, the requirement that the polynomials are homogeneous is without lost of generality as homogenization does not affect the property $Q_{k} \in \sqrt{\left(Q_{i}, Q_{j}\right)}$.

The proof follows a similar outline to the proof of Theorem 7 .

Proof of Theorem 8. Partition the polynomials in each $\mathcal{T}_{i}$ to two sets. Let $\mathcal{L}_{i}$ be the set of all squares and $Q_{i}$ be the rest. Denote $\left|Q_{i}\right|=m_{i}$.

Call a polynomial $Q \in Q_{1}$ bad for $Q_{2}$ if there are less than $m_{2} / 100$ polynomials $Q_{2} \in Q_{2}$ so that $\operatorname{span}\left\{Q, Q_{2}\right\}$ contains a polynomial from $Q_{3}$, i.e., $Q$ and $Q_{2}$ satisfy Case 1 of Theorem 29 (but not Case 2). We say that $Q \in Q_{1}$ is bad for $Q_{3}$ if the equivalent condition is satisfied. We say $Q \in Q_{1}$ is bad if it is bad for both $Q_{2}$ and $Q_{3}$. We call the polynomials in $Q_{2}, Q_{3}$ bad and good in a similar way.

We handle two cases according to whether there is at most one bad polynomial for each $Q_{i}$ or not. 


\section{AMIR SHPILKA}

\section{There is at most one bad polynomial for each $Q_{j}$ :}

In this case, in a similar fashion to the first case of Theorem 7, we get from Theorem 25 that the linear span of the polynomials in $Q:=Q_{1} \cup Q_{2} \cup Q_{3}$ has dimension $O(1)$.

As in the proof of Theorem 7 we next extend the bound to also include the linear functions in $\cup_{i} \mathcal{T}_{i}$. Assume $Q_{1}, \ldots, Q_{k}$ for some $k=O(1)$ span $Q$. We now repeat the following process. We start with $\mathcal{J}=\left\{Q_{1}, \ldots, Q_{k}\right\}$ and $V=\emptyset$. If there is some nontrivial linear combination of the polynomials in $I$ that is equal to a quadratic of the form $F(V)+a_{1} b_{1}+a_{2} b_{2}$, where $a_{i}, b_{i}$ are linear functions then we add $a_{1}, a_{2}, b_{1}, b_{2}$ to $V$ and remove one of the polynomials that participated in the linear combination from $\mathcal{J}$. We continue doing so until no such linear combination exists or until $\mathcal{J}$ is empty. At the end $|V| \leq 4 k=O(1)$. As before we abuse notation and think of $V$ as the linear space spanned by the linear functions in it.

It remains to bound the dimension of $\mathcal{L}:=\mathcal{L}_{1} \cup \mathcal{L}_{2} \cup \mathcal{L}_{3}$. We do so in a similar fashion to the proof of Claim 46. Denote $\mathcal{L}^{\prime}=\mathcal{L} \backslash V$.

First, we apply a random projection to the linear functions in $V$ so that they are all equal to some multiple of $z$. We next show that the set $\mathcal{L}^{\prime} \cup\{z\}$ satisfies the Sylvester-Gallai condition and hence its dimension is $O(1)$ as needed (we abuse notation and denote with $\mathcal{L}^{\prime}$ the projection of $\mathcal{L}^{\prime}$, which, as before, still consists of pairwise independent linear functions).

Let $x, y \in \mathcal{L}^{\prime}$ come from two different $\mathcal{L}_{i}$. Let $Q$ be such that $Q \in \sqrt{(x, y)}$. If $Q \in \mathcal{Q}$ then $Q=Q^{\prime}+G(z)$, where $Q^{\prime}$ is a linear combination of the polynomials in J. Note however, that by definition of $V, Q^{\prime}$ must be zero as otherwise we would have a linear combination of small rank and then the set J would be different. Hence, $Q=G(z)$. It follows that $z \in \operatorname{span}\{x, y\}$ and so $x, y, z$ are linearly dependent as required. If, on the other hand, $Q \in \mathcal{L}$ then $Q=\ell^{2}$ and it follows that $\ell \in \operatorname{span}\{x, y\}$. In either case, there is a third linear function in $\mathcal{L}^{\prime} \cup\{z\}$ that is spanned by $x, y$ as claimed.

Note that if $\mathcal{L} \subseteq \mathcal{L}_{i}$ for some $i$ then we easily conclude this case by picking any $x \in \mathcal{L}$ and any $Q$ in a different set and as above conclude that $x \in \operatorname{span}\{z\}$.

\section{There are at least two bad polynomial for some $Q_{j}$ :}

To ease notation assume w.l.o.g. that there are at least two bad polynomials for $Q_{3}$. The next claim gives something similar to the first part in the proof of Claim 47.

Claim 50. Assume $Q_{1}, Q_{2} \in Q_{1} \cup Q_{2}$ are bad for $Q_{3}$, then there is a space $V$ of linear functions of dimension $O(1)$ so that at least $0.98 m_{3}$ of the polynomials in $Q_{3}$ are in the linear span of $Q_{1}$ and quadratic polynomials over $V$.

Proof. Notice that for $Q_{1}$ there are $0.99 m_{3}$ polynomials in $Q^{\prime} \in Q_{3}$ that even together with $Q_{1}$ do not span any other polynomial in $Q_{2}$. The same holds for $Q_{2}$. Consider a polynomial $Q^{\prime} \in Q_{3}$ so that $Q_{1}$ and $Q^{\prime}$ do not span any other polynomial in $Q_{2}$. We conclude that $Q_{1}, Q^{\prime}$ satisfy Case 2 or Case 3 of Theorem 29. Indeed, if $Q_{1}$ and $Q^{\prime}$ satisfy item 1 of Theorem 29 then they span some polynomial in $\mathcal{L}_{2}$ and in particular they span a square of a linear function, but this means that they also satisfy Case 2 of Theorem 29. 
From the discussion above it follows that there are at least $0.98 m_{3}$ polynomials in $Q_{3}$ satisfying Case 2 or Case 3 of the theorem with $Q_{1}$ and $Q_{2}$. Let $\mathcal{F}_{3}$ be the set of these polynomials in $Q_{3}$. We partition $\mathcal{F}_{3}$ to three sets $\mathcal{J}_{3}, \mathcal{J}_{3}, \mathcal{K}_{3}$ so that those polynomials in $\mathcal{J}_{3}$ satisfy Case 3 of Theorem 29 with $Q_{1}$, those in $\mathcal{J}_{3}$ satisfy Case 3 of Theorem 29 with $Q_{2}$ and those in $\mathcal{K}_{3}$ satisfy Case 2 of Theorem 29 with both $Q_{1}$ and $Q_{2}$. As before we would like to apply Corollary 43 and Claim 44 to conclude that there is a an $O(1)$-dimensional space $V^{\prime}$ of linear functions such that all those $0.98 m_{3}$ polynomials of $\mathcal{F}_{3}$ are in the linear span of quadratics over $V^{\prime}$ and $Q_{1}$. The only problem is that the proof of Claim 44 should be tailored to the colored case, which is what we do next (indeed, Claim 38 can be applied without any changes and therefore also Corollary 43).

Note that if $Q \in Q_{1}$ satisfies Case 3 of Theorem 29 with some polynomial in $Q_{3}$ then it also satisfies the same case with a polynomial in $Q_{2}$.

Claim 51. Let $\mathrm{J}_{2} \subseteq \mathrm{Q}_{2}$ and $\mathrm{J}_{3} \subseteq Q_{3}$ be irreducible quadratics that satisfy Case 3 of Theorem 29 with an irreducible $Q \in Q_{1}$. Then, there exists an $O(1)$-dimensional space $V$ such that all polynomials in $\mathcal{J}_{2} \cup \mathcal{J}_{3}$ are quadratic polynomials in the linear functions in $V$.

We postpone the proof of the claim to subsection 6.1 and continue with the proof of Claim 50 . By applying Claim 51 first to $J_{3}$ and then to $\mathcal{J}_{3}$ we conclude that $J_{3} \cup \mathcal{J}_{3}$ are quadratics over a set of $O(1)$ linear functions $V$. Corollary 43 implies that every quadratic in $\mathcal{K}_{3}$ is in the linear span of $Q_{1}$ and quadratics over an $O(1)$-sized set $V^{\prime}$. combining $V$ and $V^{\prime}$ the claim follows. This completes the proof of Claim 50.

Let $V$ be the $O(1)$-dimensional space and $\mathcal{F}_{3} \subseteq Q_{3}$ the set of polynomials guaranteed by Claim 50 .

To continue we again have to consider two cases. The first is when there are two polynomials that are bad for $Q_{1}$ or for $Q_{2}$ (so far we assumed there are at least two bad polynomials for $Q_{3}$ ). The second case is when at most one polynomial is bad for $Q_{1}$ and at most one polynomial is bad for $Q_{2}$.

(a) There are two bad polynomials for some $Q_{i}, i \in[2]$ :

Assume w.l.o.g. that $i=2$. As before Claim 50 implies that there is a polynomial $Q_{2}$ and an $O(1)$-dimensional space $U$ such that $0.98 m_{2}$ of the polynomials in $Q_{2}$ are in the linear span of $Q_{2}$ and quadratics over $U$. Call those polynomials $\mathcal{F}_{2}$. Let $W=U+V$ be an $O(1)$-dimensional space containing both $U$ and $V$.

We now check whether there is any nontrivial linear combination of $Q_{1}$ and $Q_{2}$ that is of the form $a \cdot b+a^{\prime} \cdot b^{\prime}+F(W)$. If such a combination exists then we add $a, a^{\prime}, b, b^{\prime}$ to $W$ (and abusing notation call the new sets $W$ as well) and replace one polynomial that appeared in this combination with the other. I.e. if $Q_{2}$ appeared in such a combination then we think of the space that is spanned by $Q_{1}$ and $W$ rather than by $Q_{2}$ and $W$. We continue to do so once again if necessary. 


\section{AMIR SHPILKA}

Assume further, w.l.o.g., that $\left|Q_{2}\right| \geq\left|Q_{3}\right|$. Partition the set $Q_{1}$ to three sets $\mathcal{J}, \mathcal{J}, \mathcal{K}$ so that:

Each $Q \in \mathcal{J}$ satisfies Case 3 of Theorem 29 with at least one polynomial in $\mathcal{F}_{2}$.

Each $Q \in \mathcal{J}$ satisfies Case 2 of Theorem 29 with at least two polynomials in $\mathcal{F}_{2}$.

Each $Q \in \mathcal{K}$ satisfies Case 1 of Theorem 29 with all except possibly one polynomial in $\mathcal{F}_{2}$.

Claim 53. With the notation above we prove the following claims.

(i) The linear span of all polynomials in J has dimension $O(1)$.

(ii) All polynomials in J are polynomials over $W$.

(iii) All polynomials in $\mathcal{K}$ are in the linear span of $Q_{1}, Q_{2}$ and quadratics over $W$.

Proof. The proof of item (i) follows exactly as in Claim 51.

To show item (ii) we proceed as in the discussion following the proof of Claim 48. Consider a polynomial $Q \in \mathcal{J}$. Let $F_{1}, F_{2} \in Q_{2}$ satisfy Case 2 of Theorem 29 with $Q$. Then, after rescaling, there are $a_{1}, a_{2}$ so that $Q+a_{1}^{2}=F_{1}$ and $Q+a_{2}^{2}=F_{2}$. Hence, $a_{1}^{2}-a_{2}^{2}=F_{2}-F_{1}$. As $F_{2}-F_{1}$ is a linear combination of $Q$ and quadratics over $W$, it must be the case that $F_{2}-F_{1}$ are defined over $W$ alone as otherwise we would have replaced $Q_{2}$ with two linear functions as described above. Thus, $a_{1}^{2}-a_{2}^{2}=F(W)$ and it follows that $a_{1}, a_{2} \in W$ and hence $Q$ is a polynomial over $W$.

Finally, to prove item (iii) we note that for every $Q \in \mathcal{K}$ there are at least $0.98 m_{2}-1$ polynomials $Q_{2} \in \mathcal{F}_{2}$ so that for each of them there is $Q_{3} \in Q_{3} \cap \operatorname{span}\left\{Q, Q_{2}\right\}$. If there exists such a combination where $Q_{3} \in \mathcal{F}_{3}$ then it follows that $Q$ is a linear combination of $Q_{1}, Q_{2}$ and quadratics over $W$ (as all polynomials in $\mathcal{F}_{2}$ and $\mathcal{F}_{3}$ are). If we always get $Q_{3} \notin \mathcal{F}_{3}$ then as $\left|Q_{3} \backslash \mathcal{F}_{3}\right| \leq 0.02 m_{3} \leq 0.02 m_{2}<(1 / 2) \cdot\left|\mathcal{F}_{2}\right|$ there exist $Q_{2}, Q_{2}^{\prime} \in \mathcal{F}_{2}$ and $Q_{3} \in Q_{3}$ so that $Q_{3} \in \operatorname{span}\left\{Q, Q_{2}\right\}, \operatorname{span}\left\{Q, Q_{2}^{\prime}\right\}$. As every two polynomials in our set are linearly independent this implies that $Q \in \operatorname{span}\left\{Q_{2}, Q_{2}^{\prime}\right\}$, and in particular it is in the span of $Q_{2}$ and quadratics over $W$, as claimed.

A similar argument will now show that $Q_{2}$ and $Q_{3}$ are also contained in an $O(1)$-dimensional space. We thus showed that there is an $O(1)$-dimensional space containing all polynomials in $Q_{1} \cup Q_{2} \cup Q_{3}$. It remains to bound the dimension of the linear functions in $\mathcal{L}_{1} \cup \mathcal{L}_{2} \cup \mathcal{L}_{3}$. This can be done at exactly the same way as before. This concludes the proof of Theorem 8 in this case.

(b) At most one polynomial is bad for $Q_{1}$ and at most one polynomial is bad for $Q_{2}$

In this case we reduce to the extended robust Edelstein-Kelly theorem (Theorem 26).

For each $i \in[2]$ partition $Q_{i}$ to $\mathcal{J}_{i}, \mathcal{J}_{i}$ and $\mathcal{K}_{i}$ as in Equation 52 except that we now consider $\mathcal{F}_{3}$ instead of $\mathcal{F}_{2}$ when partitioning. It follows, exactly as in the proof of Claim 53, that there is an $O(1)$-dimensional space $U$ that all polynomials in $\mathcal{J}_{1} \cup \mathcal{J}_{1} \cup \mathcal{J}_{2} \cup \mathcal{J}_{2}$ are in the linear span of $Q_{1}$ and quadratics over $U$.

Let $W$ be the space spanned by $Q_{1}$ and quadratics over $U$. Clearly $\operatorname{dim}(W)=O(1)$. 
For $i \in[2]$ let $\mathcal{K}_{i}^{\prime} \subset \mathcal{K}_{i}$ be those polynomials in $\mathcal{K}_{i}$ that are not in $W$. Similarly, define $K_{3}^{\prime} \subset \Omega_{3}$. Let $W_{i}=W \cap Q_{i}$, for $i \in[3]$.

We now observe that the sets $Q_{1}=\mathcal{K}_{1}^{\prime} \cup W_{1}, Q_{2}=\mathcal{K}_{2}^{\prime} \cup W_{2}, Q_{3}=\mathcal{K}_{3}^{\prime} \cup W_{3}$ satisfy the conditions in the statement of Theorem 26 (where the $\mathcal{K}_{i}$ in the statement of the theorem is our $\mathcal{K}_{i}^{\prime}$ ), with parameters $r=O(1), c=2$ and $\delta=1 / 100$, when we identify our quadratic polynomials with their vectors of coefficients.

Indeed, as we are in the case where there is at most one bad polynomial for $Q_{1}$ and at most one bad polynomial for $2_{2}$ we see that there are at most 2 "exceptional" vectors defined that way. Furthermore, from the definition of $\mathcal{K}_{1}^{\prime}, \mathcal{K}_{2}^{\prime}$ (Equation 52) no point in them is "exceptional" when considering $Q_{3}$.

Thus, Theorem 26 guarantees the existence of a space $Y$ of dimension $O_{c}\left(r+1 / \delta^{3}\right)=O(1)$ that spans all vectors in the set $Q_{1} \cup Q_{2} \cup Q_{3}$. We are almost done - we still have to deal with the linear function in $\mathcal{L}_{1} \cup \mathcal{L}_{2} \cup \mathcal{L}_{3}$. This however is done exactly as before.

This completes the proof of Theorem 8 (modulo the proof of Claim 51 that we give next).

\subsection{Missing proof}

In this section we give the proof of Claim 51. For convenience we repeat the statement of the claim.

Claim (Claim 51). Let $\mathrm{J}_{2} \subseteq Q_{2}$ and $\mathrm{J}_{3} \subseteq Q_{3}$ be irreducible quadratics that satisfy Case 3 of Theorem 29 with an irreducible $Q \in Q_{1}$. Then, there exists an $O(1)$-dimensional space $V$ such that all polynomials in $J_{2} \cup J_{3}$ are quadratic polynomials in the linear functions in $V$.

Proof of Claim 51. Let $\mathcal{J}_{2}=\left\{F_{i}\right\}_{i}$ and $\mathcal{J}_{3}=\left\{G_{i}\right\}_{i}$. As before we take $V^{\prime}$ to be the space spanned by the linear functions in a minimal representation of $Q$. Clearly $\operatorname{dim}\left(V^{\prime}\right) \leq 4$. Let $z$ be a new variable. Set each basis element of $V^{\prime}$ to a random multiple of $z$ (as before, we pick the multiples independently, uniformly at random from $[0,1])$. Each $F_{i}, G_{i}$ now becomes $z \cdot b_{i}$ for some nonzero $b_{i}$. Indeed, if we further set $z=0$ then all linear functions in the representation of $Q$ vanish and hence $F_{i}$ and $G_{i}$ also vanish. ${ }^{11}$ Furthermore, for any $i \neq j, b_{i}$ and $b_{j}$ are linearly independent (as in Claim 45), unless they both equal to multiples of $z$.

Let $J_{1}$ be the set of quadratics in $Q_{1}$ that after making the restriction become quadratics of the form $z \cdot b$. Clearly $Q_{1}$ is such a polynomial.

We next show that the linear functions $\left\{b_{i}\right\}_{i} \cup\{z\}$, where the $b_{i}$ are the linear functions coming from $\mathcal{J}_{1} \cup \mathcal{J}_{2} \cup \mathcal{J}_{3}$ as described above, satisfy the usual Sylvester-Gallai condition and conclude by Theorem 15 that their rank is $O(1)$.

Claim 54. If some polynomial in $\mathrm{J}_{2}\left(\mathrm{~J}_{3}\right)$ is projected to $z \cdot b$ where $b$ is linearly independent of $z$ then there is some polynomial in $\mathrm{J}_{3}\left(\mathrm{~J}_{2}\right)$ that is projected to $z \cdot c$ for some c linearly independent of $z$.

Proof. Consider any polynomial $Q^{\prime} \in \mathrm{J}_{2}$ that was projected to $z \cdot b$, where $b$ is linearly independent of $z$, and let $Q^{\prime \prime} \in \mathcal{J}_{3}$ be in $\sqrt{\left(Q_{1}, Q^{\prime}\right)}$. Assume for a contradiction that $Q^{\prime \prime}$ was projected to $z^{2}$. Claim 45 implies that if this is the case then all linear functions in a minimal representation of $Q^{\prime \prime}$ belong to $V^{\prime}$.

\footnotetext{
${ }^{11}$ Here too we use the fact that $Q$ is irreducible and hence the two linear functions that make $F_{i}$ (or $G_{i}$ ) vanish appear in $V^{\prime}$ (Claim 14).
} 


\section{AMIR SHPILKA}

We thus have that $Q^{\prime \prime}=Q^{\prime \prime}\left(V^{\prime}\right)$. We can also assume w.l.o.g. that $Q^{\prime}=Q^{\prime}\left(V^{\prime}, b\right)$ (by completing $V^{\prime} \cup\{b\}$ to a basis for the entire space of linear functions and projecting the other basis elements to random multiples of $b$ ). We next show that $Q^{\prime \prime} \in \sqrt{Q_{1}}$, which implies $Q^{\prime \prime}$ is a multiple of $Q_{1}$ in contradiction.

We again resort to Theorem 29. It is clear that $Q^{\prime \prime} \notin \operatorname{span}\left\{Q_{1}, Q^{\prime}\right\}$. So we are left with the two other cases.

1. $Q_{1}$ and $Q^{\prime}$ span a square of a linear function: It is not hard to see that in this case we must have (after rescaling) that $Q^{\prime}=b^{2}+\ell\left(V^{\prime}\right) \cdot b+A^{\prime}\left(V^{\prime}\right)$. Consider any assignment to $V^{\prime}$ that makes $Q_{1}$ vanish. Clearly there is a value to $b$ that also makes $Q^{\prime}$ vanish for that assignment. Thus $Q^{\prime \prime}$ also vanishes. Therefore, any assignment that makes $Q_{1}=0$ also makes $Q^{\prime \prime}=0$ which is what we wanted to prove.

2. There are two linear functions $v_{1}, v_{2} \in V^{\prime}$ so that $Q_{1}, Q^{\prime}, Q^{\prime \prime} \in \sqrt{\left(v_{1}, v_{2}\right)}$ : Denote $Q_{1}=v_{1} \cdot u_{1}+v_{2}$. $u_{2}, Q^{\prime}+v_{1} \cdot b_{1}+v_{2} \cdot b_{2}$ and $Q^{\prime \prime}=v_{1} \cdot w_{1}+v_{2} \cdot w_{2}$, where $w_{i}, u_{i} \in V^{\prime}$. Project $v_{1}$ and $v_{2}$ to random multiples of a new variable $y$. Then, our new polynomials are now $Q_{1}=y \cdot u, Q^{\prime}=y \cdot b^{\prime}$ and $Q^{\prime \prime}=y \cdot w$, where $u, w \in V^{\prime}$ (where we abuse notation and refer to the projection of $V^{\prime}$ also as $V^{\prime}$ ) and, with probability $1, b^{\prime} \notin V^{\prime}$. Consider the assignment $u=b^{\prime}=0$. It follows that we also get $y \cdot w=0$. However, as $y, w, u \in V^{\prime}$ and $b^{\prime} \notin V^{\prime}$ it must be the case that $y \cdot w=0$ modulo $u$. Thus, after this projection we get that $Q^{\prime \prime} \in \sqrt{\left(Q_{1}\right)}$. This implies however that $Q^{\prime \prime}$ is a multiple of $Q_{1}$ as it cannot be the case that $Q_{1}$ was projected to a square (as this would imply that it was only a function of $v_{1}$ and $v_{2}$ and hence a reducible polynomial). Claim 45 implies that this was also the case before the projection, in contradiction.

We continue with the proof of Claim 51. Claim 54 establishes that either all polynomials in $\mathcal{J}_{2} \cup \mathcal{J}_{3}$ were projected to $z^{2}$ or that both $J_{2}$ and $J_{3}$ contain polynomials that were projected to quadratics of the form $z \cdot b$ where $b$ is linearly independent of $z$.

We are now ready to show that the linear functions $\left\{b_{i}\right\}_{i} \cup\{z\}$, where $b_{i}$ are the linear functions in $\mathcal{J}_{1} \cup \mathcal{J}_{2} \cup \mathcal{J}_{3}$, satisfy the usual Sylvester-Gallai condition.

Consider any two quadratics $A_{2}=z \cdot b_{2} \in \mathcal{J}_{2}, A_{3}=z \cdot b_{3} \in \mathcal{J}_{3}$ so that neither $b_{2}$ nor $b_{3}$ is a multiple of $z$. If $b_{2}$ and $b_{3}$ span $z$ then we are done. So assume that $z \notin \operatorname{span}\left\{b_{2}, b_{3}\right\}$. Let $A_{1}$ vanish when $A_{2}, A_{3}$ vanish. Then clearly $z$ divides $A_{1}$. Thus $A_{1}=z \cdot b_{1}$ is in $\mathcal{J}_{1}$ and so $b_{1}$ is in our set. Further, when we set $b_{2}=b_{3}=0$ both $A_{2}, A_{3}$ vanish and hence also $A_{1}$ vanishes. Since $z \notin \operatorname{span}\left\{b_{1}, b_{2}\right\}$ this implies that $b_{1} \in \operatorname{span}\left\{b_{2}, b_{3}\right\}$ and so in this case $b_{2}$ and $b_{3}$ span a third linear function in our set. Note also that by Claim $45 b_{1}$ is not a multiple of $b_{2}$ nor of $b_{3}$ as this would imply that $A_{1}$ and $A_{2}$ (or $A_{3}$ ) are linearly dependent in contradiction to our assumption.

This argument shows that whenever $b_{2}$ and $b_{3}$ are not a multiple of $z$ (and they come from different sets), the set $\left\{b_{i}\right\}_{i} \cup\{z\}$ contains a nontrivial linear combination of them. In a similar fashion to Corollary 16 and Theorem 26 we get that the dimension of all those linear functions is $O(1) .{ }^{12}$

\footnotetext{
${ }^{12}$ We note that we cannot apply Theorem 26 as is as it may be the case that $z$ appears in all three sets. However, it is not hard to see that a small modification of it will capture this case as well.
} 
As in the proof of Claim 44 we repeat this argument again for a different random mapping to multiples of $z$ and conclude in the same way that every polynomial in $J_{2} \cup J_{3}$ is a polynomial over some $O(1)$-dimensional space $V$.

This completes the proof of Claim 51.

\section{Conclusions and future research}

In this work we proved analogs of theorems of Sylvester-Gallai and Edelstein-Kelly for quadratic polynomials. These results directly relate to the problem of obtaining deterministic algorithms for testing identities of $\Sigma^{[3]} \Pi^{[d]} \Sigma \Pi^{[2]}$ circuits. As mentioned in section 1 in order to obtain PIT algorithms we need even stronger extensions of these results - something in the line of Conjecture 4 that was proposed by Gupta [Gup14].

It is quite likely that Theorems 7 and 8 could be extended to obtain a positive answer to Conjecture 4 for $r=2$ and $k=3$. Indeed, there is an analog of Theorem 10 that suits the condition of the conjecture (for $r=2$ and $k=3$ ). Peleg [Pel19] used this extension of Theorem 10 to generalize Theorem 7 to the case where for every $Q_{i}$ and $Q_{j}$ it holds that whenever they vanish the product of the other $Q_{k}$ 's vanishes as well. This is a significant step towards resolving Conjecture 4 (for $r=2$ and $k=3$ ).

However, extending our approach to the case of more than 3 multiplication gates (or more than 3 sets as in Theorem 8) seems more challenging. Indeed, the structure theorem gets more complicated in the sense that there are many more cases to consider and it seems unlikely that a similar approach will work for "higher values of 3". Similarly, while proving a structural theorem for degree 3 polynomials is possible, it seems that extending the exact same approach to significantly higher degrees may be less easy. Thus, we believe that a different proof approach may be needed in order to obtain PIT algorithms for $\Sigma^{[O(1)]} \Pi^{[d]} \Sigma \Pi^{[O(1)]}$ circuits.

Another interesting question is, stated vaguely, understanding the conditions under which we get a Sylvester-Gallai kind of behavior. By now many variants of the theorem are known: The original

Sylvester-Gallai theorem, the colored version of it (Edelstein-Kelly theorem), robust versions of it (by [BDWY13, DSW14]), extensions to subspaces [DH16], $k$-wise dependencies [Han65, BDWY13], our results for quadratic polynomials and more. It is an intriguing question whether there is a common generalization of all these cases or some framework that contain all these different results.

\section{Acknowledgments}

I would like to thank Shir Peleg for helpful discussions and Ankit Gupta for commenting on an earlier version of the paper. I also thank the anonymous reviewer for their comments. Part of this work was done while the author was visiting NYU.

\section{References}

[Agr05] Manindra Agrawal. Proving Lower Bounds Via Pseudo-random Generators. In Ramaswamy Ramanujam and Sandeep Sen, editors, FSTTCS 2005: Foundations of Software Technology and 


\section{AMIR SHPILKA}

Theoretical Computer Science, 25th International Conference, Hyderabad, India, December 15-18, 2005, Proceedings, volume 3821 of Lecture Notes in Computer Science, pages 92-105. Springer, 2005. 2

[BDWY13] Boaz Barak, Zeev Dvir, Avi Wigderson, and Amir Yehudayoff. Fractional Sylvester-Gallai theorems. Proceedings of the National Academy of Sciences, 110(48):19213-19219, 2013. 2, 5, 6, $7,8,12,31$

[BM90] Peter Borwein and William O. J. Moser. A survey of Sylvester's problem and its generalizations. Aequationes Mathematicae, 40:111-135, 1990. 2

[BMS13] Malte Beecken, Johannes Mittmann, and Nitin Saxena. Algebraic independence and blackbox identity testing. Inf. Comput., 222:2-19, 2013. 3, 4

[CKS18] Chi-Ning Chou, Mrinal Kumar, and Noam Solomon. Hardness vs Randomness for Bounded Depth Arithmetic Circuits. In Rocco A. Servedio, editor, 33rd Computational Complexity Conference, CCC 2018, June 22-24, 2018, San Diego, CA, USA, volume 102 of LIPIcs, pages 13:1-13:17. Schloss Dagstuhl - Leibniz-Zentrum fuer Informatik, 2018. 2

[CLO07] David A. Cox, John Little, and Donal O'Shea. Ideals, Varieties, and Algorithms: An Introduction to Computational Algebraic Geometry and Commutative Algebra. Springer, 3rd edition, 2007. 7,8

[DH16] Zeev Dvir and Guangda Hu. Sylvester-Gallai for Arrangements of Subspaces. Discrete \& Computational Geometry, 56(4):940-965, 2016. 31

[DS07] Zeev Dvir and Amir Shpilka. Locally Decodable Codes with Two Queries and Polynomial Identity Testing for Depth 3 Circuits. SIAM J. Comput., 36(5):1404-1434, 2007. 2

[DSW14] Zeev Dvir, Shubhangi Saraf, and Avi Wigderson. Improved rank bounds for design matrices and a new proof of Kelly's theorem. Forum of Mathematics, Sigma, 2, 2014. Pre-print available at arXiv:1211.0330. 2, 5, 6, 7, 8, 12, 31

[DSY09] Zeev Dvir, Amir Shpilka, and Amir Yehudayoff. Hardness-Randomness Tradeoffs for Bounded Depth Arithmetic Circuits. SIAM J. Comput., 39(4):1279-1293, 2009. 2

[EK66] Michael Edelstein and Leroy M. Kelly. Bisecants of finite collections of sets in linear spaces. Canadian Journal of Mathematics, 18:375-280, 1966. 9

[FGT19] Stephen A. Fenner, Rohit Gurjar, and Thomas Thierauf. A deterministic parallel algorithm for bipartite perfect matching. Commun. ACM, 62(3):109-115, 2019. 2

[For14] Michael A. Forbes. Polynomial identity testing of read-once oblivious algebraic branching programs. $\mathrm{PhD}$ thesis, Massachusetts Institute of Technology, 2014. 2 
SYLVESTER-GALLAI TYPE THEOREMS FOR QUADRATIC POLYNOMIALS

[FS13] Michael A. Forbes and Amir Shpilka. Explicit Noether Normalization for Simultaneous Conjugation via Polynomial Identity Testing. In Prasad Raghavendra, Sofya Raskhodnikova, Klaus Jansen, and José D. P. Rolim, editors, Approximation, Randomization, and Combinatorial Optimization. Algorithms and Techniques - 16th International Workshop, APPROX 2013, and 17th International Workshop, RANDOM 2013, Berkeley, CA, USA, August 21-23, 2013. Proceedings, volume 8096 of Lecture Notes in Computer Science, pages 527-542. Springer, 2013. 2

[FSV18] Michael A. Forbes, Amir Shpilka, and Ben Lee Volk. Succinct Hitting Sets and Barriers to Proving Lower Bounds for Algebraic Circuits. Theory of Computing, 14(1):1-45, 2018. 2

[GT17] Rohit Gurjar and Thomas Thierauf. Linear matroid intersection is in quasi-NC. In Hamed Hatami, Pierre McKenzie, and Valerie King, editors, Proceedings of the 49th Annual ACM SIGACT Symposium on Theory of Computing, STOC 2017, Montreal, QC, Canada, June 19-23, 2017, pages 821-830. ACM, 2017. 2

[Gup14] Ankit Gupta. Algebraic Geometric Techniques for Depth-4 PIT \& Sylvester-Gallai Conjectures for Varieties. Electronic Colloquium on Computational Complexity (ECCC), 21:130, 2014. 1, 3, 4, 31

[Han65] Sten Hansen. A generalization of a theorem of Sylvester on the lines determined by a finite point set. Mathematica Scandinavica, 16:175-180, 1965. 31

[HS80] Joos Heintz and Claus-Peter Schnorr. Testing Polynomials which Are Easy to Compute (Extended Abstract). In Proceedings of the 12th annual STOC, pages 262-272, 1980. 2

[KI04] Valentine Kabanets and Russell Impagliazzo. Derandomizing Polynomial Identity Tests Means Proving Circuit Lower Bounds. Computational Complexity, 13(1-2):1-46, 2004. 2

[KS09a] Zohar Shay Karnin and Amir Shpilka. Reconstruction of Generalized Depth-3 Arithmetic Circuits with Bounded Top Fan-in. In Proceedings of the 24th Annual IEEE Conference on Computational Complexity, CCC 2009, Paris, France, 15-18 July 2009, pages 274-285. IEEE Computer Society, 2009. 2

[KS09b] Neeraj Kayal and Shubhangi Saraf. Blackbox Polynomial Identity Testing for Depth 3 Circuits. In 50th Annual IEEE Symposium on Foundations of Computer Science, FOCS 2009, October 25-27, 2009, Atlanta, Georgia, USA, pages 198-207. IEEE Computer Society, 2009. 2

[KSS15] Swastik Kopparty, Shubhangi Saraf, and Amir Shpilka. Equivalence of Polynomial Identity Testing and Polynomial Factorization. Computational Complexity, 24(2):295-331, 2015. 2

[MU05] Michael Mitzenmacher and Eli Upfal. Probability and Computing - Randomized Algorithms and Probabilistic Analysis. Cambridge University Press, 2005. 7

[Mul17] Ketan D. Mulmuley. Geometric complexity theory V: Efficient algorithms for Noether normalization. J. Amer. Math. Soc., 30(1):225-309, 2017. 2 


\section{AMIR SHPILKA}

[Pel19] Shir Peleg. Sylvester-Gallai type theorem for quadratic polynomials. Master's thesis, Tel Aviv University, 2019. 31

[Sax09] Nitin Saxena. Progress on polynomial identity testing. Bulletin of EATCS, 99:49-79, 2009. 2

[Sax 14] Nitin Saxena. Progress on Polynomial Identity Testing-II. In M. Agrawal and V. Arvind, editors, Perspectives in Computational Complexity: The Somenath Biswas Anniversary Volume, Progress in Computer Science and Applied Logic, pages 131-146. Springer International Publishing, 2014. 2

[Shp09] Amir Shpilka. Interpolation of Depth-3 Arithmetic Circuits with Two Multiplication Gates. SIAM J. Comput., 38(6):2130-2161, 2009. 2

[Sin16] Gaurav Sinha. Reconstruction of Real Depth-3 Circuits with Top Fan-In 2. In Ran Raz, editor, 31st Conference on Computational Complexity, CCC 2016, May 29 to June 1, 2016, Tokyo, Japan, volume 50 of LIPIcs, pages 31:1-31:53. Schloss Dagstuhl - Leibniz-Zentrum fuer Informatik, 2016. 2

[SS12] Nitin Saxena and C. Seshadhri. Blackbox Identity Testing for Bounded Top-Fanin Depth-3 Circuits: The Field Doesn't Matter. SIAM J. Comput., 41(5):1285-1298, 2012. 2

[SS13] Nitin Saxena and C. Seshadhri. From Sylvester-Gallai configurations to rank bounds: Improved blackbox identity test for depth-3 circuits. $J . A C M, 60(5): 33,2013.2$

[ST17] Ola Svensson and Jakub Tarnawski. The Matching Problem in General Graphs Is in Quasi-NC. In Chris Umans, editor, 58th IEEE Annual Symposium on Foundations of Computer Science, FOCS 2017, Berkeley, CA, USA, October 15-17, 2017, pages 696-707. IEEE Computer Society, 2017. 2

[SV18] Shubhangi Saraf and Ilya Volkovich. Black-Box Identity Testing of Depth-4 Multilinear Circuits. Combinatorica, 38(5):1205-1238, 2018. 3

[SY10] Amir Shpilka and Amir Yehudayoff. Arithmetic Circuits: A survey of recent results and open questions. Foundations and Trends in Theoretical Computer Science, 5(3-4):207-388, 2010. 2

\section{AUTHOR}

Amir Shpilka

Professor

Tel Aviv University

Tel Aviv, Israel

shpilka@tauex.tau.ac.il

https://www.cs.tau.ac.il/ shpilka 